Article

\title{
Exploiting the Nucleophilicity of the Nitrogen Atom of Imidazoles: One-Pot Three-Component Synthesis of Imidazo-Pyrazines
}

\author{
Ubaldina Galli $^{1}$, Rejdia Hysenlika ${ }^{1}$, Fiorella Meneghetti ${ }^{2}$ (D) Erika Del Grosso ${ }^{1}{ }^{(D)}$ \\ Sveva Pelliccia $^{3}$, Ettore Novellino ${ }^{3}$, Mariateresa Giustiniano ${ }^{3, *}$ (D) and Gian Cesare Tron $1, * \mathbb{D}$ \\ 1 Dipartimento di Scienze del Farmaco, Università del Piemonte Orientale “A. Avogadro", 28100 Novara, Italy; \\ ubaldina.galli@uniupo.it (U.G.); 10026282@studenti.uniupo.it (R.H.); erika.delgrosso@uniupo.it (E.D.G.) \\ 2 Dipartimento di Scienze Farmaceutiche, Università degli Studi di Milano, 20133 Milano, Italy; \\ fiorella.meneghetti@unimi.it \\ 3 Dipartimento di Farmacia, Università degli Studi di Napoli “Federico II", 80131 Napoli, Italy; \\ sveva.pelliccia@unina.it (S.P.); ettore.novellino@unina.it (E.N.) \\ * Correspondence: mariateresa.giustiniano@unina.it (M.G.); giancesare.tron@uniupo.it (G.C.T.)
}

Received: 5 May 2019; Accepted: 20 May 2019; Published: 21 May 2019

\begin{abstract}
A novel one-pot multicomponent reaction to synthesize substituted imidazopyrazines is described. In brief, $1 H$-(imidazol-5-yl)- $N$-substituted methanamines react with aldehydes and isocyanides in methanol at room temperature to give imidazopyrazine derivatives in excellent yields. The imidazole nitrogen atom was able to intercept the nascent nitrilium ion, channeling the reaction toward to the sole formation of imidazopyrazines, suppressing the competitive formation of other possible side products deriving from the reaction with the high-energy nitrilium ion. The number of examples and the variability of the nature of isocyanides, aldehydes, and amine components herein employed, witness the robustness of this novel methodology.
\end{abstract}

Keywords: multicomponent reactions; interrupted Ugi reactions; isocyanides

\section{Introduction}

Although multistep synthesis is the only possible choice for the preparation of highly complex natural products, it is important to keep in mind the actual feasibility and pragmatic aspects of those synthetic sequences. A recent paper [1] demonstrated that for the famous 49 steps Woodward's synthesis of chlorophyll a, $1 \mathrm{mg}$ of product would have required $230 \mathrm{~kg}$ of ethyl acetoacetate, the first chemical used in that project. Therefore, it is important, for concepts like "efficiency of synthesis" [2] and "reaction mass efficiency" [3], to consider the real practical application of any synthetic project. For this reason, the identification of novel reactions enabling the one-pot assembly of compounds of medium structural complexity is an important field of research with significant implications for all chemistry branches [4]. Multicomponent reactions (MCRs) are among these types of reactions, allowing for the formation of three or four covalent bonds in one single chemical operation with a net reduction of time and chemical waste. When providing the desired chemical output, MCRs usually represent the most efficient choice available to chemists [5-15].

Two more advantages of these MCRs are the rapid generation of a library of compounds, and the possibility to generate molecular scaffolds, which are difficult or in some cases impossible to obtain by the classical two-component chemistry [16]. Finally, when compared to a multistep synthesis, the excellence of a multicomponent reaction can be easily and rapidly ascertained by a large number of chemists shortly after publication. Characteristics such as yield, ease of purification, and versatility can be easily proved, and their scope expanded, beyond the original design $[17,18]$. 
One of the most important and versatile multicomponent reactions available to chemists is the Ugi reaction [8], a four-component one-pot reaction among equimolar amounts of amines (1), aldehydes (2), isocyanides (3), and carboxylic acids (5) which yields $\alpha$-acylaminoamides (6) under mild reaction conditions in an alcoholic solvent (Scheme 1).

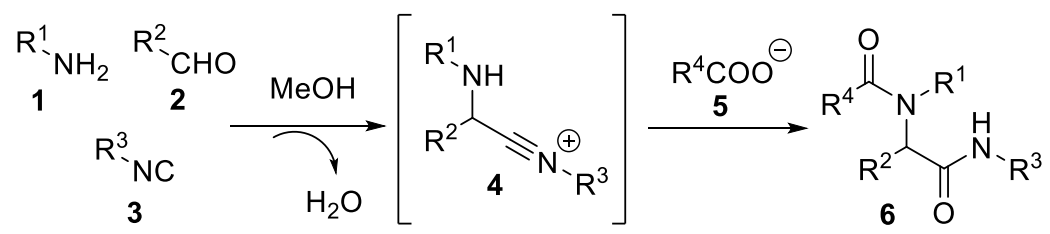

Scheme 1. The Ugi reaction.

Thanks to its versatility, this reaction can also be used as the conceptual starting point for the discovery of novel MCRs, for example, by replacing one of the components with a suitable isostere [19]. Another possibility consists in removing the carboxylic acid, allowing the nitrilium ion intermediate (4) to be intramolecularly intercepted by a passive nucleophile unable to interfere with the initial elementary steps of the Ugi reaction. This strategy is known as interrupted Ugi reaction and, despite its intrinsic potentiality, it has not been fully exploited by the scientific community [20-28]. The interrupted Ugi reaction could be a very powerful tool to create sets of multi-functionalized and drug-like heterocycles, as exemplified in Figure 1.<smiles>[R]Nc1nc2ccc[R]c2nc1[R]</smiles><smiles>[R2]Nc1c([R7])[nH]c2ccccc12</smiles><smiles>[R]C1C(=O)OCCN1[R]</smiles><smiles>[R]C1C(=[18O])N([R7])CCN1[R]</smiles><smiles>[R1]C1([R1])NCCCN=C1NP</smiles><smiles>[R7]Nc1oc(C([R7])NC(=O)NCCCC)nc1[R]</smiles>

Figure 1. Examples of heterocycles obtained through an interrupted Ugi reaction.

Herein, we would like to disclose a novel interrupted Ugi reaction, exploiting for the first time the imidazole ring, as a soft nucleophile able to intramolecularly intercept the nascent nitrilium ion. This intermediate enables the formation of otherwise synthetically challenging substituted imidazopyrazines, in excellent yields and under mild reaction conditions (r.t. in $\mathrm{MeOH}$ ) Multicomponent reactions represent a powerful chemical tool to expedite medicinal chemistry and early drug discovery programs [29], especially when combined with automation and flow synthesis [30-32].

\section{Results and Discussion}

To perform this task, we synthesized the corresponding imidazole $N$-substituted methanamines via reductive amination between formylimidazole derivatives (7-9) and primary amines (Scheme 2), preparing eight different building blocks (13-20) (Figure 2). 


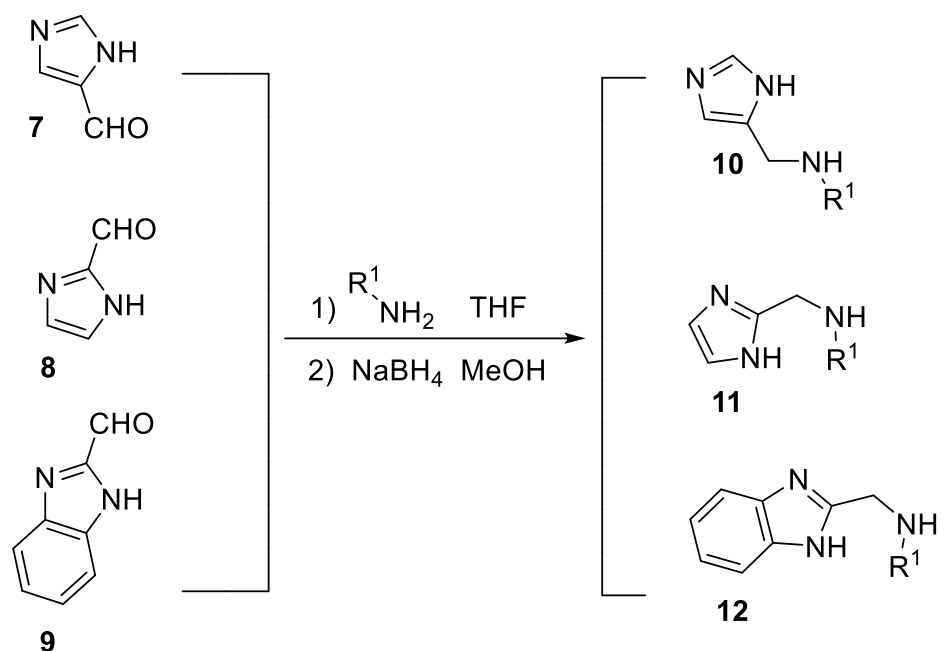

Scheme 2. Synthesis of imidazole $N$-substituted methanamines.

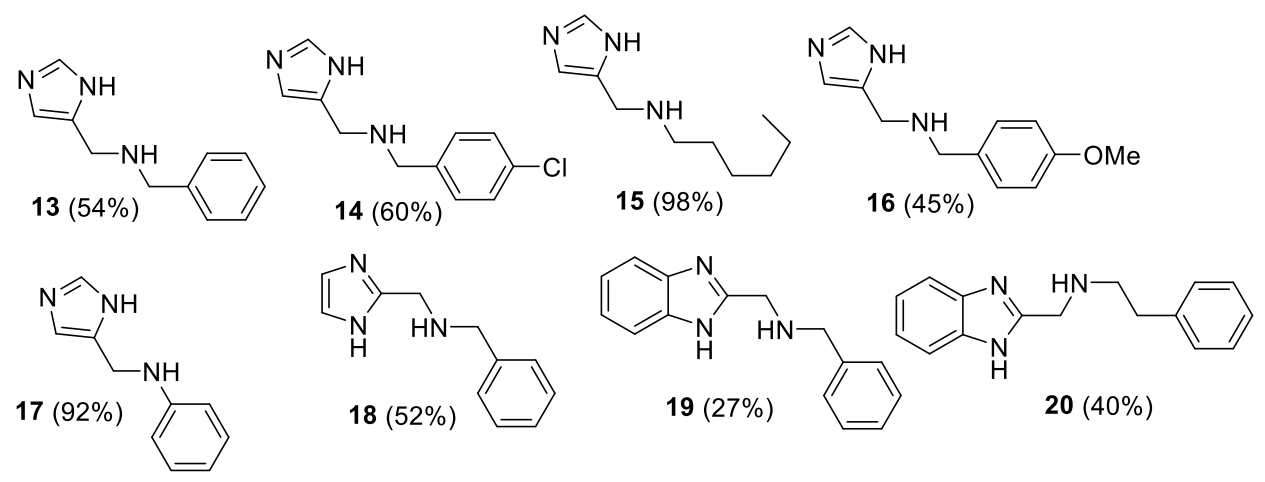

Figure 2. Synthesized imidazole $N$-substituted methanamines.

With the different imidazole $N$-substituted methanamines, we then carried out the MCR using twelve different isocyanides (21-32) and twelve aldehydes (33-44) in methanol, at room temperature overnight (Figure 3).

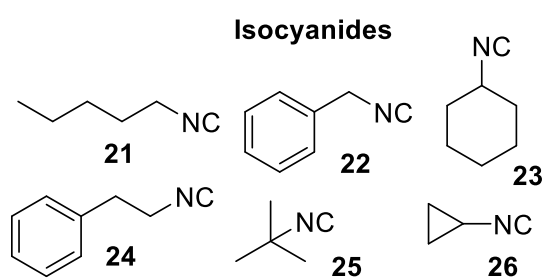

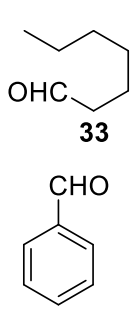

36<smiles>COc1ccc(C=O)cc1</smiles>

40

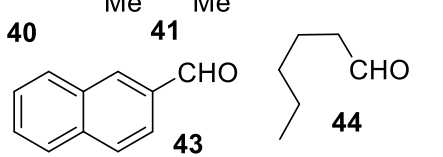

Figure 3. Isocyanides and aldehydes used. 
To our delight, all the attempted reactions consistently resulted in the formation of the desired imidazopyrazines (45-80), usually in excellent yields, without the formation of any by-products (Figure 4).

In detail, yields ranged from $95 \%$ to $25 \%$ and showed to be unaffected by the nature of isocyanides. Indeed, we were able to successfully use all kinds of isocyanides: aromatic, primary, secondary, and tertiary aliphatic, and isocyanoacetate, without noting a significant decrease in yield. Aliphatic and aromatic aldehydes containing either electron-withdrawing or electron-donating groups reacted in similar ways. A reduction in yield was observed above all with heterocyclic aldehydes $\mathbf{3 5}$ and $\mathbf{3 7}$ (Figure 3). Except for compounds 77 and 78, which were obtained as a mixture of isomers, we have generally observed the formation of one single geometrical isomer for the amidines. To further confirm this data, an X-ray crystallographic analysis of compound 79 was also performed (Figure 5).

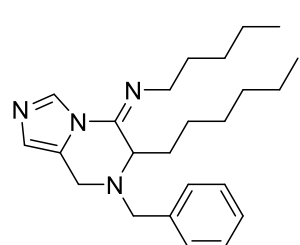

$45(75 \%)$
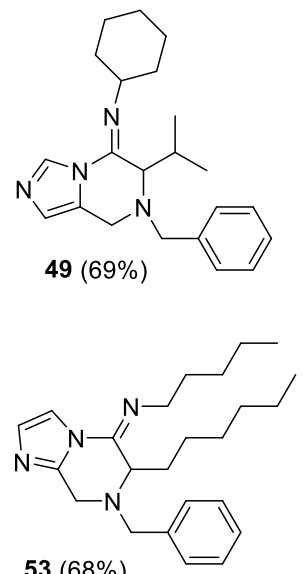

$53(68 \%)$

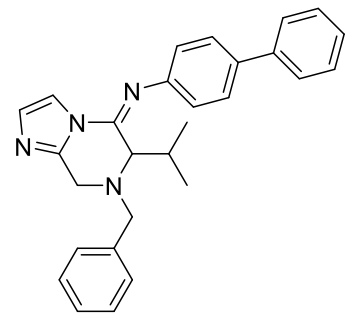

$57(70 \%)$

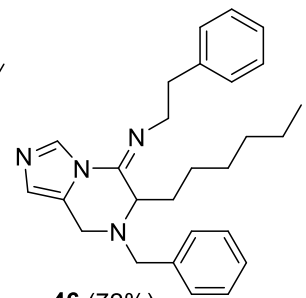

$46(72 \%)$
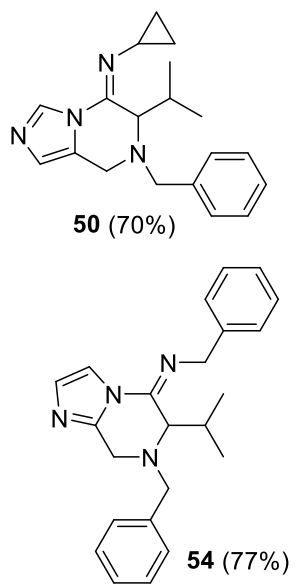

$47(55 \%)$<smiles>[Z15]C(C)(C)CC1C(CCCCCC)C(=NCc2ccccc2)n2cncc2CN1Cc1ccccc1</smiles><smiles>CC(C)C1C(=NCCc2ccccc2)n2cncc2CN1Cc1ccccc1</smiles>

$51(61 \%)$<smiles>CCCCCCC1/C(=N\C(C)(C)C)n2cncc2CN1Cc1ccccc1</smiles>

$48(79 \%)$<smiles>COC(=O)CN=C1C(C(C)C)N(Cc2ccccc2)Cc2cncn21</smiles>

$52(54 \%)$
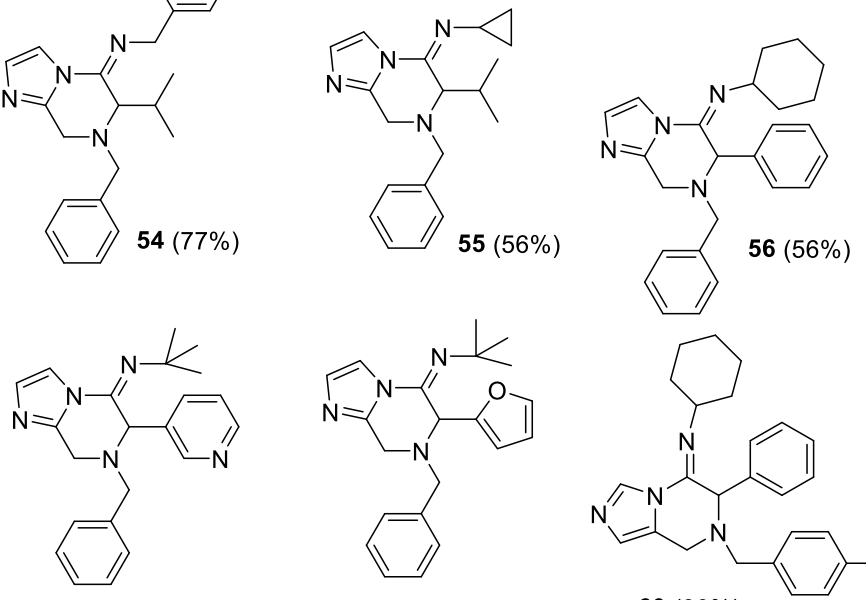

$58(45 \%)$

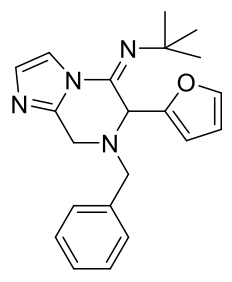

$59(50 \%)$

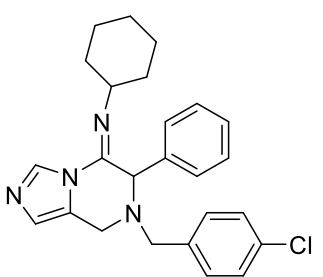

$60(80 \%)$

Figure 4. Cont. 


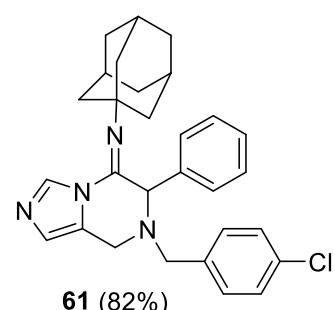<smiles>CCCCCCC1C(=NCC(=O)OC)n2cncc2CN1Cc1ccc(Cl)cc1</smiles><smiles>CCCCCCC1C(=NC(C)(C)C)N(Cc2ccccc2)Cc2nc3ccccc3n2C1=NC(C)(C)C</smiles><smiles>CCCCCN=C1C(c2ccc(Cl)cc2)N(Cc2ccccc2)Cc2nc3ccccc3n21</smiles><smiles>CCCCCCC1/C(=N/C2CCCCC2)n2c(nc3ccccc32)CN1Cc1ccccc1</smiles>

$66(59 \%)$<smiles>CCCCCCN1Cc2ncn(CCc3ccccc3)c2C(=NCC(=O)OC)C1c1cncn1C=NC(C)(C)C</smiles>

$67(65 \%)$

$68(85 \%)$
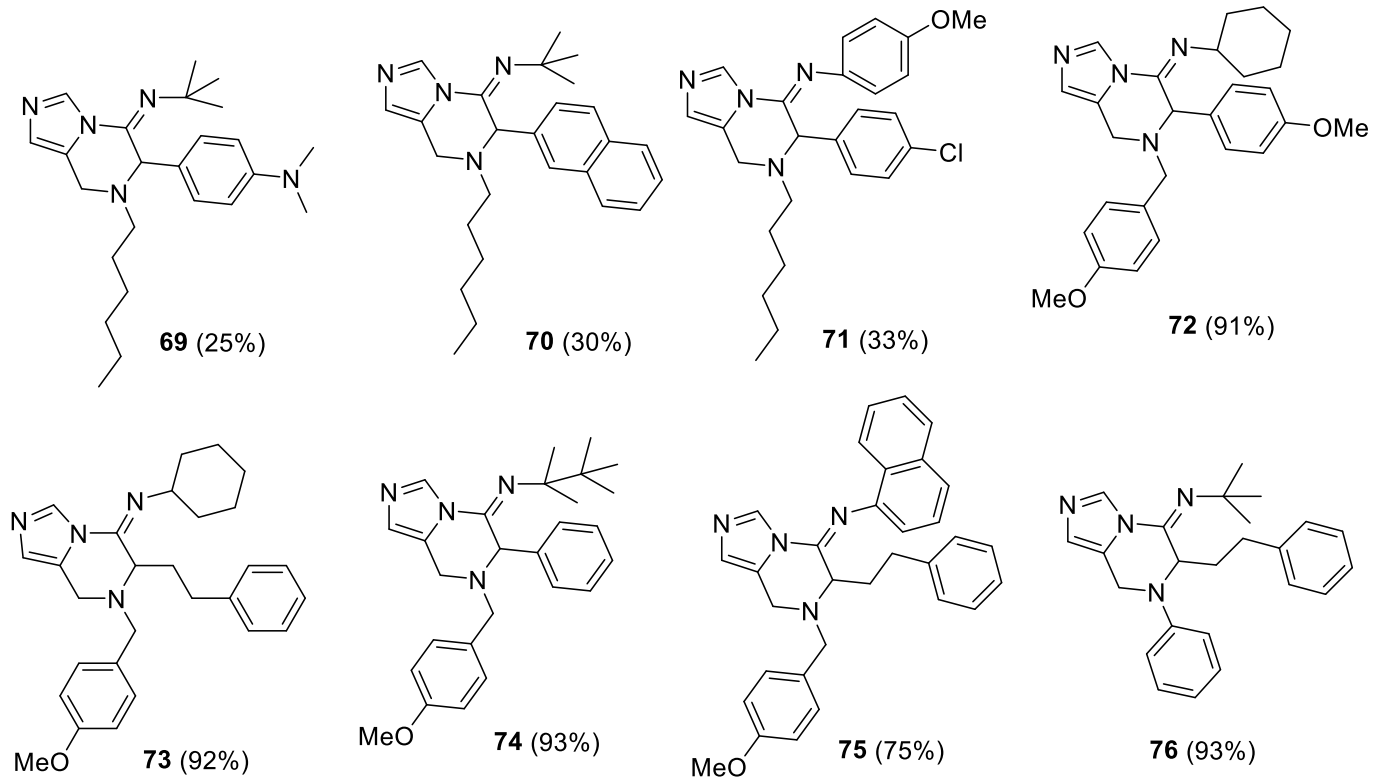

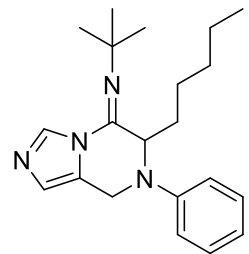

$77(95 \%)$

isomeric mixture

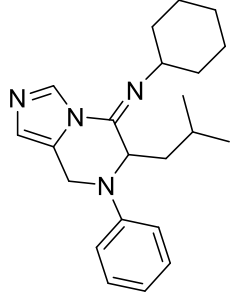

$78(90 \%)$ isomeric mixture

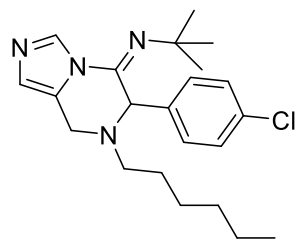

$79(71 \%)$
$76(93 \%)$

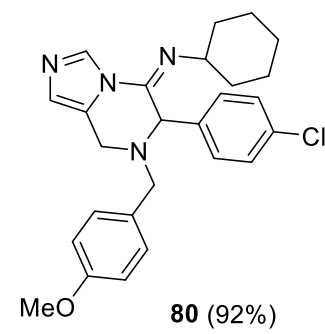

Figure 4. Synthesized imidazopyrazines. 


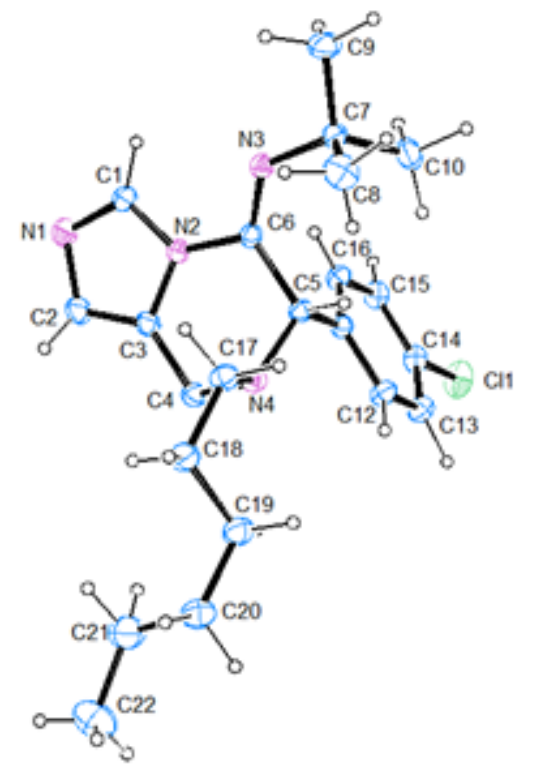

Figure 5. ORTEP [33] view of $\mathbf{7 9}$ and the relative arbitrary atom-numbering scheme (thermal ellipsoids at $40 \%$ probability).

The crystallographic data showed that the amidine adopts an E configuration about the $\mathrm{C} 6=\mathrm{N} 3$ double bond; the piperazine ring is characterized by an almost half-chair conformation (Cremer-Pople puckering parameters [34] are: QT $=0.428(1) \AA, \theta=46.3(5)^{\circ}$ and $\left.\varphi=-160(1)^{\circ}\right)$. The chloro-benzene substituent is in axial conformation and it is perpendicularly oriented with respect to the imidazole moiety (dihedral angle of $89(1)^{\circ}$ ). The bicyclic core presents a certain degree of electron delocalization, as shown by the shortening of the N2-C6 1.434(2) $\AA$ and C3-C4 1.498(2) $\AA$ bond lengths. The conformation of N4-side chain is mainly characterized by the torsional angles $\omega 1 \mathrm{C} 4-\mathrm{N} 4-\mathrm{C} 17-\mathrm{C} 18$ of $60.8(1)^{\circ}, \omega 2$ N4-C17-C18-C19 of 53.4(1) ${ }^{\circ}$, and $\omega 3$ C18-C19-C20-C21 of 63.6(1) .

In the light of these results, in Scheme 3 we propose the following reaction mechanism: the secondary amine A reacts with the carbonyl compound $\mathbf{B}$ in methanol, forming the iminium ion $\mathbf{C}$ after the loss of a molecule of water [35]. The iminium ion cannot undergo intramolecular cyclization through the nitrogen atom of imidazole, being disfavored by an incorrect orbital overlapping [36], thus allowing for the isocyanide $\mathbf{D}$ to react, forming the highly energetic nitrilium ion $\mathbf{E}$. A fast and irreversible 6-exo-dig intramolecular trapping by means of the nitrogen atom of imidazole affords compound F. As suggested by one of the reviewers as many 5-endo-trig processes, although unfavoured, are observed, it could be possible that the putative aminal formed via a 5-endo-trig cyclization may undergo reopening and can be irreversibly trapped by the isocyanide. Note that the nitrogen atom attacks the nitrilium ion in a stereoselective way [37,38], affording the amidines in $Z$ configuration. Finally, due to a peri interaction, compound $\mathbf{F}$ adopts a stable $E$ configuration $\mathbf{G}$ by nitrogen inversion. 


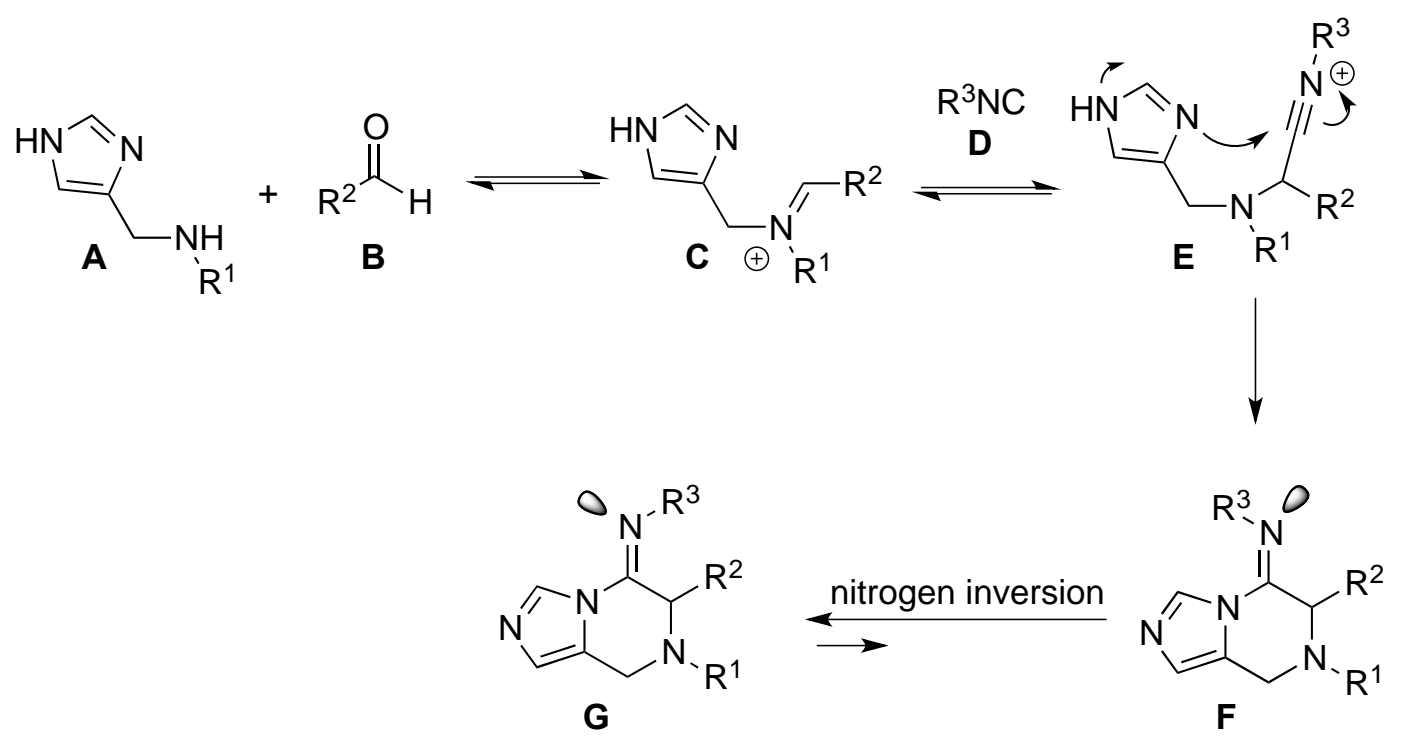

Scheme 3. Proposed reaction mechanism.

\section{Materials and Methods}

\subsection{Solvents and Reagents}

Commercially available solvents and reagents were used without further purification. Tetrahydrofuran (THF) was distilled immediately before use from $\mathrm{Na}$ /benzophenone under a slight positive atmosphere of dry nitrogen. When needed, the reactions were performed in oven-dried glassware under a positive pressure of dry nitrogen.

\subsection{Chromatography}

Column chromatography was performed on silica gel (Merck Kieselgel 60, 230-400 mesh ASTM) using the indicated eluents. Thin layer chromatography (TLC) was carried out on $5 \times 20 \mathrm{~cm}$ plates with a layer thickness of 0.25 mm (Merck Silica gel 60 F254, Merck KGaA, Darmstadt, Germany). When necessary they were visualized using $\mathrm{KMnO} 4$ reagent or Dragendorff reagent.

\subsection{Spectra}

Infrared spectra were recorded on an FT-IR Thermo-Nicolet Avatar spectrometer with absorption maxima ( $v$ max) recorded in wavenumbers $\left(\mathrm{cm}^{-1}\right)$. NMR spectra were recorded using a JEOL ECP $300 \mathrm{MHz}$ spectrometer. Chemical shifts $(\delta)$ are quoted in parts per million referenced to the residual solvent peak. The multiplicity of each signal is designated using the following abbreviations: s, singlet; $\mathrm{d}$, doublet; $\mathrm{t}$, triplet; q, quartet; quint, quintet; sext, sextet; hept, heptet; m, multiplet; br s, broad singlet. Coupling constants $(\mathrm{J})$ are reported in Hertz $(\mathrm{Hz})$ (for more data see Supplementary Materials). Mass spectra were recorded on a Thermo Finningan LCQ-deca XP-plus mass spectrometer (Waltham, Massachusetts, USA) equipped with an ESI source and an ion trap detector. Melting points were determined using a Stuart Scientific SMP3 apparatus and remain uncorrected.

\subsection{Preparation of Secondary Amines (13-20)}

The appropriate imidazolecarboxaldehyde derivative (10 $\mathrm{mmol}, 1$ equiv.) was dissolved in methanol. The amine (1 equiv.) in dry THF (5.0 mL), and activated $4 \AA$ molecular sieves (2.00 g) were added. The reaction mixture was stirred at room temperature, under nitrogen for $12 \mathrm{~h}$. Then, sodium borohydride (1.2 equiv.) was added portionwise and the reaction was stirred at room temperature. After $3 \mathrm{~h}$ water was added, and the mixture was stirred for $10 \mathrm{~min}$. Molecular sieves were filtered off through a Celite pad. The filtrate was evaporated under reduced pressure. The residue was taken 
up with EtOAc and the organic layer was washed with $2 \mathrm{~N} \mathrm{NaOH}(\times 1)$, dried over sodium sulfate and concentrated in vacuo. The crude material was purified by column chromatography using the indicated eluents.

N-((1H-imidazol-5-yl)methyl)-1-phenylmethanamine (13). The crude material was purified by column chromatography (EtOAc/MeOH 90:10 $+1 \% \mathrm{NH}_{4} \mathrm{OH}$ ) to give the product as a white solid (1.01 g, yield 54\%). ${ }^{1} \mathrm{H}-\mathrm{NMR}\left(300 \mathrm{MHz}, \mathrm{CD}_{3} \mathrm{OD}\right) \delta 7.61(\mathrm{~s}, 1 \mathrm{H}), 7.30-7.22(\mathrm{~m}, 5 \mathrm{H}), 6.97(\mathrm{~s}, 1 \mathrm{H}), 3.72(\mathrm{br}$ $\mathrm{s}, 4 \mathrm{H}) ;{ }^{13} \mathrm{C}-\mathrm{NMR}\left(75 \mathrm{MHz}, \mathrm{CD}_{3} \mathrm{OD}\right) \delta 139.3,135.8,135.2,128.4,128.3,127.0,117.1,52.4,44.4 ; \mathrm{IR}(\mathrm{KBr})$ $3551,3411,3235,2842,1564,1492,1454,1322,1085,1066 v_{\max } / \mathrm{cm}^{-1}$; m.p. 103-104 ${ }^{\circ} \mathrm{C}$; MS (ESI) $\mathrm{m} / \mathrm{z}$ Calcd for $\mathrm{C}_{11} \mathrm{H}_{14} \mathrm{~N}_{3}{ }^{+}$: 188.1183 ; found: $188.1184[\mathrm{M}+\mathrm{H}]^{+}$.

$\mathrm{N}$-((1H-imidazol-5-yl)methyl)-1-(4-chlorophenyl)methanamine (14). The crude material was purified by column chromatography $\left(\mathrm{EtOAc} / \mathrm{MeOH} 90: 10+1 \% \mathrm{NH}_{4} \mathrm{OH}\right)$ to give the product as yellow oil (1.33 g, yield 60\%). ${ }^{1} \mathrm{H}-\mathrm{NMR}\left(300 \mathrm{MHz}, \mathrm{CD}_{3} \mathrm{OD}\right) \delta 7.63(\mathrm{~s}, 1 \mathrm{H}), 7.33-7.28(\mathrm{~m}, 4 \mathrm{H}), 6.99(\mathrm{~s}$, $1 \mathrm{H}), 3.71(\mathrm{~s}, 2 \mathrm{H}), 3.70(\mathrm{~s}, 2 \mathrm{H}) ;{ }^{13} \mathrm{C}-\mathrm{NMR}\left(75 \mathrm{MHz}, \mathrm{CD}_{3} \mathrm{OD}\right) \delta 139.5,137.1,136.6,134.1,131.3,129.7,118.5$, 53.0, 46.0; IR (neat) 3089, 2841, 1490, 1452, 1089, 1014, 936, 802, $623 v_{\mathrm{max}} / \mathrm{cm}^{-1}$; MS (ESI) $\mathrm{m} / z$ Calcd for $\mathrm{C}_{11} \mathrm{H}_{13} \mathrm{ClN}_{3}{ }^{+}$: 222.0793; found: $222.0795[\mathrm{M}+\mathrm{H}]^{+}$.

$\mathrm{N}$-((1H-imidazol-5-yl)methyl)hexan-1-amine (15). The crude material was purified by column chromatography (dichloromethane/ methanol 90:10) to give the product as a yellowish oil (1.77 $\mathrm{g}, 98 \%$ yield). ${ }^{1} \mathrm{H}$ NMR (400 MHz, CD $\left.{ }_{3} \mathrm{OD}\right) \delta 7.61(\mathrm{~s}, 1 \mathrm{H}), 6.97(\mathrm{~s}, 1 \mathrm{H}), 3.70(\mathrm{~s}, 2 \mathrm{H}), 2.58-2.55(\mathrm{~m}, 2 \mathrm{H}), 1.52-1.47$ $(\mathrm{m}, 2 \mathrm{H}), 1.33-1.30(\mathrm{~m}, 6 \mathrm{H}), 0.91-0.88(\mathrm{~m}, 3 \mathrm{H}) ;{ }^{13} \mathrm{C} \mathrm{NMR}\left(100 \mathrm{MHz}, \mathrm{CD}_{3} \mathrm{OD}\right) \delta 135.1,134.8,117.0,48.2$, 44.6, 31.4, 28.5, 26.6, 22.2, 13.0. IR (neat) 3088, 2925, 2855, 1466, 1089, 822, $626 v_{\max } / \mathrm{cm}^{-1}$; MS (ESI) $\mathrm{m} / \mathrm{z}$ Calcd for $\mathrm{C}_{10} \mathrm{H}_{20} \mathrm{~N}_{3}{ }^{+}$: 182.1652; found: $182.1655[\mathrm{M}+\mathrm{H}]^{+}$.

$\mathrm{N}$-((1H-imidazol-5-yl)methyl)-1-(4-methoxyphenyl)methanamine (16). The crude material was purified by column chromatography (dichloromethane/ methanol 90:10) to give the product as a yellowish oil $(0.977 \mathrm{~g}, 45 \%$ yield $) .{ }^{1} \mathrm{H}$ NMR $\left(400 \mathrm{MHz}, \mathrm{CD}_{3} \mathrm{OD}\right) \delta 7.62(\mathrm{~s}, 1 \mathrm{H}), 7.23(\mathrm{~d}, J=8.4 \mathrm{~Hz}, 2 \mathrm{H})$, $6.98(\mathrm{~s}, 1 \mathrm{H}), 6.86(\mathrm{~d}, J=8.4 \mathrm{~Hz}, 2 \mathrm{H}), 3.76(\mathrm{~s}, 3 \mathrm{H}), 3.70(\mathrm{~s}, 2 \mathrm{H}), 3.67(\mathrm{~s}, 2 \mathrm{H}) ;{ }^{13} \mathrm{C} \mathrm{NMR}\left(100 \mathrm{MHz}, \mathrm{CD}_{3} \mathrm{OD}\right)$ $\delta$ 158.7, 135.7, 135.3, 131.5, 129.5, 117.5, 113.8, 55.2, 52.4, 45.0. IR (neat) 3117, 2836, 1611, 1512, 1456, $1244,1089,1032,815,626 v_{\max } / \mathrm{cm}^{-1}$; MS (ESI) $\mathrm{m} / \mathrm{z}$ Calcd for $\mathrm{C}_{12} \mathrm{H}_{16} \mathrm{~N}_{3} \mathrm{O}^{+}: 218.1288$; found: 218.1291 $[\mathrm{M}+\mathrm{H}]^{+}$.

$\mathrm{N}$-((1H-imidazol-5-yl)methyl)aniline (17). The crude material was purified by column chromatography (dichloromethane/ methanol 98:2) to give the product as a yellowish oil $(1.59 \mathrm{~g}, 92 \%$ yield). ${ }^{1} \mathrm{H}$ NMR $\left(400 \mathrm{MHz}, \mathrm{CD}_{3} \mathrm{OD}\right) \delta 7.58(\mathrm{~s}, 1 \mathrm{H}), 7.10-7.06(\mathrm{~m}, 2 \mathrm{H}), 6.93(\mathrm{~s}, 1 \mathrm{H}), 6.67-6.59(\mathrm{~m}, 3 \mathrm{H})$, $4.23(\mathrm{~s}, 2 \mathrm{H}) ;{ }^{13} \mathrm{C} \mathrm{NMR}\left(100 \mathrm{MHz}, \mathrm{CD}_{3} \mathrm{OD}\right) \delta 148.5,128.5,117.0,112.9,40.4$. IR (neat) $3388,3049,2815$, 2632, 1605, 1505, 1317, 1002, 836, $750 v_{\max } / \mathrm{cm}^{-1}$; Mp 127-128 ${ }^{\circ} \mathrm{C}$; MS (ESI) $\mathrm{m} / z$ Calcd for $\mathrm{C}_{10} \mathrm{H}_{12} \mathrm{~N}_{3}{ }^{+}$: 174.1026; found: $174.1028[\mathrm{M}+\mathrm{H}]^{+}$.

$\mathrm{N}$-((1H-imidazol-2-yl)methyl)-1-phenylmethanamine (18). The crude material was purified by column chromatography (EtOAc/MeOH 95:5 + 1\% NH $\mathrm{NH}_{4} \mathrm{OH}$ and $\left.\mathrm{EtOAc} / \mathrm{MeOH} 90: 10+1 \% \mathrm{NH}_{4} \mathrm{OH}\right)$ to give the product as yellow oil $(0.972 \mathrm{~g}$, yield $52 \%) .{ }^{1} \mathrm{H}-\mathrm{NMR}\left(300 \mathrm{MHz}, \mathrm{CD}_{3} \mathrm{OD}\right) \delta 7.26-7.18(\mathrm{~m}, 5 \mathrm{H})$, $6.97(\mathrm{~s}, 2 \mathrm{H}), 3.78(\mathrm{~s}, 2 \mathrm{H}), 3.66(\mathrm{~s}, 2 \mathrm{H}) ;{ }^{13} \mathrm{C}-\mathrm{NMR}\left(75 \mathrm{MHz}, \mathrm{CD}_{3} \mathrm{OD}\right) \delta 147.9,140.4,129.4(2 \mathrm{C}), 128.2,122.7$, 53.8, 46.3; IR (neat) 2919, 1452, 1098, 986, 855, 736, $697 v_{\max } / \mathrm{cm}^{-1}$; MS (ESI) $\mathrm{m} / z$ Calcd for $\mathrm{C}_{11} \mathrm{H}_{14} \mathrm{~N}_{3}{ }^{+}$: 188.1183; Found: $188.1184[\mathrm{M}+\mathrm{H}]^{+}$.

$\mathrm{N}$-(1H-benzo[d]imidazol-2-yl)methyl)-1-phenylmethanamine (19). The crude material was purified by column chromatography (Pet/EtOAc 10:90) to give the product as yellow solid ( $0.640 \mathrm{~g}$, yield $27 \%$ ). ${ }^{1} \mathrm{H}-\mathrm{NMR}\left(300 \mathrm{MHz}, \mathrm{CD}_{3} \mathrm{OD}\right) \delta$ 7.54-7.51 (m, 2H), 7.36-7.19 (m, 7H), $4.01(\mathrm{~s}, 2 \mathrm{H}), 3.81(\mathrm{~s}, 2 \mathrm{H}) ;{ }^{13} \mathrm{C}-\mathrm{NMR}$ (75 MHz, CD $\left.{ }_{3} \mathrm{OD}\right) \delta$ 155.0, 140.6, 129.4, 128.2, 123.4, 115.6, 54.1, 47.0; IR (neat) 3555, 3414, 3028, 2838, $1618,1437,1269,1024,750 v_{\max } / \mathrm{cm}^{-1}$; m.p. $143-145^{\circ} \mathrm{C}$; MS (ESI) $\mathrm{m} / z$ Calcd for $\mathrm{C}_{15} \mathrm{H}_{16} \mathrm{~N}_{3}{ }^{+}$: 238.1339; found: $238.1339[\mathrm{M}+\mathrm{H}]^{+}$. 
$\mathrm{N}$-((1H-benzo[d]imidazol-2-yl)methyl)-2-phenylethanamine (20). The crude material was purified by column chromatography (Pet/EtOAc 20:80 and EtOAc/MeOH 90:10) to give the product as yellow solid (1.00 g, yield 40\%). ${ }^{1} \mathrm{H}-\mathrm{NMR}\left(300 \mathrm{MHz}, \mathrm{CD}_{3} \mathrm{OD}\right) \delta$ 7.53-7.49 (m, 2H), 7.26-7.12 (m, 7H), $4.01(\mathrm{~s}$, $2 \mathrm{H}), 2.92-2.87\left(\mathrm{~m}, \mathrm{~A}_{2} \mathrm{~B}_{2}\right.$ system, $\left.2 \mathrm{H}\right), 2.83-2.78\left(\mathrm{~m}, \mathrm{~A}_{2} \mathrm{~B}_{2}\right.$ system, $\left.2 \mathrm{H}\right) ;{ }^{13} \mathrm{C}-\mathrm{NMR}\left(75 \mathrm{MHz}, \mathrm{CD}_{3} \mathrm{OD}\right) \delta$ 154.9, 141.0, 129.6, 129.5, 127.2, 123.5, 115.7, 51.7, 47.5, 36.9; IR (neat) 3554, 3415, 2950, 2817, 1618, 1537, $1453,1424,1275,1125,1025,751,704 v_{\max } / \mathrm{cm}^{-1}$; m.p. 141-143 ${ }^{\circ} \mathrm{C}$; MS (ESI) $\mathrm{m} / z$ Calcd for $\mathrm{C}_{16} \mathrm{H}_{18} \mathrm{~N}_{3}{ }^{+}$: 252.1496; Found: $252.1497[\mathrm{M}+\mathrm{H}]^{+}$.

\subsection{Synthesis of Substituted Imidazopyrazines and Benzoimidazopyrazines (45-80)}

The secondary amine $(0.5 \mathrm{mmol}, 1$ equiv. $)$ was dissolved in methanol $(2.0 \mathrm{~mL})$. Aldehyde ( $0.5 \mathrm{mmol}, 1$ equiv.) and isocyanide $(0.5 \mathrm{mmol}, 1$ equiv.) were added and the reaction was stirred at room temperature under nitrogen for $18 \mathrm{~h}$. The reaction mixture was concentrated under reduced pressure and the crude material was purified by column chromatography.

(E)-N-(7-benzyl-6-hexyl-7,8-dihydroimidazo[1,5- $a$ ]pyrazin-5(6H)-ylidene)pentan-1-amine

(45).

The crude material was purified by column chromatography (Pet/EtOAc 90:10 and Pet/EtOAc 70:30) to give the product as orange oil (153 mg, yield 75\%). ${ }^{1} \mathrm{H}-\mathrm{NMR}\left(300 \mathrm{MHz}, \mathrm{CDCl}_{3}\right) \delta 8.32(\mathrm{~s}, 1 \mathrm{H}), 7.29-7.27$ $(\mathrm{m}, 5 \mathrm{H}), 6.78(\mathrm{~s}, 1 \mathrm{H}), 4.13(\mathrm{~d}, J=17.1 \mathrm{~Hz}, \mathrm{AB}$ system, $1 \mathrm{H}), 3.80-3.68(\mathrm{~m}, 3 \mathrm{H}), 3.53(\mathrm{~d}, J=13.2 \mathrm{~Hz}, \mathrm{AB}$ system, 1H), 3.24-3.22 (m, 2H), 1.78-1.16 (m, 16H), $1.06-0.88(\mathrm{~m}, 6 \mathrm{H}) ;{ }^{13} \mathrm{C}-\mathrm{NMR}\left(75 \mathrm{MHz}, \mathrm{CDCl}_{3}\right) \delta$ 150.0, 137.9, 132.8, 128.9, 128.5, 127.7, 124.9, 124.6, 58.9, 54.8, 48.3, 40.7, 31.8, 30.9, 29.7, 28.8, 25.9, 22.7, 22.6, 14.1. IR (neat) 2953, 2927, 2857, 1671, 1461, 1347, 1093, 923, 818, $699 v_{\max } / \mathrm{cm}^{-1}$; MS (ESI) $\mathrm{m} / \mathrm{z}$ Calcd for $\mathrm{C}_{24} \mathrm{H}_{37} \mathrm{~N}_{4}{ }^{+}$: 381.3013; Found: $381.3006[\mathrm{M}+\mathrm{H}]^{+}$.

(E)-N-(7-benzyl-6-hexyl-7,8-dihydroimidazo[1,5-a]pyrazin-5(6H)-ylidene)-2-phenylethanamine (46). The crude material was purified by column chromatography (Pet/EtOAc 90:10) to give the product as orange oil $(160 \mathrm{mg}$, yield $72 \%) .{ }^{1} \mathrm{H}-\mathrm{NMR}\left(300 \mathrm{MHz}, \mathrm{CDCl}_{3}\right) \delta 8.38,(\mathrm{~s}, 1 \mathrm{H}), 7.31-7.17(\mathrm{~m}$, $10 \mathrm{H}), 6.79(\mathrm{~s}, 1 \mathrm{H}), 4.07(\mathrm{~d}, J=16.8 \mathrm{HZ}, \mathrm{AB}$ system, $1 \mathrm{H}), 3.75-3.47(\mathrm{~m}, 5 \mathrm{H}), 3.25(\mathrm{~d}, J=13.2 \mathrm{~Hz}, \mathrm{AB}$ system, $1 \mathrm{H}), 3.04-2.84(\mathrm{~m}, 2 \mathrm{H}), 1.68-1.48(\mathrm{~m}, 2 \mathrm{H}), 1.28-1.14(\mathrm{~m}, 8 \mathrm{H}), 0.89-0.83(\mathrm{~m}, 3 \mathrm{H}) ;{ }^{13} \mathrm{C}-\mathrm{NMR}(75$ $\left.\mathrm{MHz}, \mathrm{CDCl}_{3}\right) \delta 150.7,140.0,138.0,135.4,129.2,128.8,128.6,128.5,127.6,126.4,125.1,124.6,58.7,55.1$, 50.3, 40.4, 37.8, 31.8, 28.8 (2C), 25.9, 22.7, 14.2. IR (neat) 2925, 2856, 1671, 1460, 1347, 1089, 923, 744, 698 $v_{\text {max }} / \mathrm{cm}^{-1}$; MS (ESI) $\mathrm{m} / z$ Calcd for $\mathrm{C}_{27} \mathrm{H}_{35} \mathrm{~N}_{4}^{+}$: 415.2857; found: $415.2853[\mathrm{M}+\mathrm{H}]^{+}$.

(E)-N-(7-benzyl-6-hexyl-7,8-dihydroimidazo[1,5-a]pyrazin-5(6H)-ylidene)-1-phenylmethanamine (47). The crude material was purified by column chromatography (Pet/EtOAc 70:30) to give the product as orange oil (118 mg, yield 55\%). ${ }^{1} \mathrm{H}-\mathrm{NMR}\left(300 \mathrm{MHz}, \mathrm{CDCl}_{3}\right) \delta 8.41,(\mathrm{~s}, 1 \mathrm{H}), 7.33-7.23$ $(\mathrm{m}, 10 \mathrm{H}), 6.81(\mathrm{~s}, 1 \mathrm{H}), 4.52(\mathrm{~s}, 2 \mathrm{H}), 4.17(\mathrm{~d}, J=16.8 \mathrm{~Hz}, \mathrm{AB}$ system, $1 \mathrm{H}), 3.91-3.71(\mathrm{~m}, 3 \mathrm{H}), 3.54(\mathrm{~d}$, $J=12.8 \mathrm{~Hz}, \mathrm{AB}$ system, $1 \mathrm{H}), 1.82-1.20(\mathrm{~m}, 10 \mathrm{H}), 0.97-0.89(\mathrm{~m}, 3 \mathrm{H}) ;{ }^{13} \mathrm{C}-\mathrm{NMR}\left(75 \mathrm{MHz}, \mathrm{CDCl}_{3}\right) \delta 151.4$, 139.5, 137.8, 133.0, 129.0, 128.6, 127.8. 127.5, 127.1, 125.2, 124.6, 59.0, 55.1, 51.8, 40.7, 31.8, 28.8 (2C), 26.0, 22.7, 14.2; IR (neat) 2926, 1671, 1460, 1346, 1213, 1086, 922, 734, $697 v_{\max } / \mathrm{cm}^{-1}$; MS (ESI) $\mathrm{m} / \mathrm{z}$ Calcd for $\mathrm{C}_{26} \mathrm{H}_{33} \mathrm{~N}_{4}^{+}$: 401.2700 ; found: $401.2699[\mathrm{M}+\mathrm{H}]^{+}$.

(E)-N-(7-benzyl-6-hexyl-7,8-dihydroimidazo[1,5-a]pyrazin-5(6H)-ylidene)-2-methylpropan-2-amine (48). The crude material was purified by column chromatography (Pet/EtOAc 95:5 and Pet/EtOAc 70:30) to give the product as brown amorphous solid (154 mg, yield 79\%). ${ }^{1} \mathrm{H}-\mathrm{NMR}\left(300 \mathrm{MHz}, \mathrm{CDCl}_{3}\right)$ $\delta 8.26(\mathrm{~s}, 1 \mathrm{H}), 7.29-7.26(\mathrm{~m}, 5 \mathrm{H}), 6.71(\mathrm{~s}, 1 \mathrm{H}), 4.08(\mathrm{~d}, J=17.1 \mathrm{~Hz}, \mathrm{AB}$ system, $1 \mathrm{H}), 3.93-3.89(\mathrm{~m}, 1 \mathrm{H})$, 3.70-3.65 (m, 3H), 1.84-1.21 (m, 19H), 1.10-0.90 (m, 3H); ${ }^{13} \mathrm{C}-\mathrm{NMR}\left(75 \mathrm{MHz}, \mathrm{CDCl}_{3}\right) \delta 146.2,138.1$, $133.1,128.4(2 \mathrm{C}), 127.4,124.9,124.5,58.5$ (2C), 54.5, 39.6, 31.7, 31.5, 29.7, 28.8, 26.0, 22.6, 14.0; IR (KBr) 2958, 2924, 2858, 1670, 1455, 1344, 1198, 1095, 738, $652 v_{\max } / \mathrm{cm}^{-1}$; MS (ESI) $\mathrm{m} / z$ Calcd for $\mathrm{C}_{23} \mathrm{H}_{35} \mathrm{~N}_{4}{ }^{+}$: 367.2857; found: fragment ion (loss of 2-methyl-N-methylenepropan-2-amine moiety): $284.2121[\mathrm{M}+\mathrm{H}]^{+}$.

(E)-N-(7-benzyl-6-isopropyl-7,8-dihydroimidazo[1,5-a]pyrazin-5(6H)-ylidene)cyclohexanamine (49). The crude material was purified by column chromatography (Pet/EtOAc 70:30) to give the product as yellow solid (121 mg, yield 69\%). ${ }^{1} \mathrm{H}-\mathrm{NMR}\left(300 \mathrm{MHz}, \mathrm{CDCl}_{3}\right) \delta 8.26(\mathrm{~s}, 1 \mathrm{H}), 7.30-7.25(\mathrm{~m}$, 
$5 \mathrm{H}), 6.72(\mathrm{~s}, 1 \mathrm{H}), 4.11(\mathrm{~d}, J=17.4 \mathrm{~Hz}, \mathrm{AB}$ system, $1 \mathrm{H}), 3.71-3.66(\mathrm{~m}, 3 \mathrm{H}), 3.49(\mathrm{~d}, J=10.7 \mathrm{~Hz}, \mathrm{AB}$ system, $1 \mathrm{H}), 3.29-3.30(\mathrm{~m}, 1 \mathrm{H}), 1.95-1.92(\mathrm{~m}, 1 \mathrm{H}), 1.79-1.45(\mathrm{~m}, 6 \mathrm{H}), 1.24-1.20(\mathrm{~m}, 7 \mathrm{H}), 0.93(\mathrm{~d}, J=6.7 \mathrm{~Hz}, 3 \mathrm{H})$; ${ }^{13} \mathrm{C}-\mathrm{NMR}\left(75 \mathrm{MHz}, \mathrm{CDCl}_{3}\right) \delta$ 146.6, 138.2, 132.9, 128.5 (2C), 127.5, 125.3, 124.5, 62.5, 59.0, 57.4, 40.2, 34.7, 33.8, 27.9, 25.6, 24.4, 24.3, 20.2, 20.0; IR (KBr) 3033, 2922, 2850, 1672, 1618, 1455, 1340, 1245, 1214, 1090, $815 v_{\max } / \mathrm{cm}^{-1}$; m.p. $154-155^{\circ} \mathrm{C}$; MS (ESI) $\mathrm{m} / \mathrm{z}$ Calcd for $\mathrm{C}_{22} \mathrm{H}_{31} \mathrm{~N}_{4}{ }^{+}$: 351.2544; found: fragment ion (loss of $\mathrm{N}$-methylenecyclohexanamine moiety): found: $242.1653[\mathrm{M}+\mathrm{H}]^{+}$.

(E)-N-(7-benzyl-6-isopropyl-7,8-dihydroimidazo[1,5-a]pyrazin-5(6H)-ylidene)cyclopropanamine

(50). The crude material was purified by column chromatography (Pet/EtOAc 70:30) to give the product as yellow solid (108 mg, yield 70\%). ${ }^{1} \mathrm{H}-\mathrm{NMR}\left(300 \mathrm{MHz}, \mathrm{CDCl}_{3}\right) \delta 8.20(\mathrm{~s}, 1 \mathrm{H}), 7.30-7.25(\mathrm{~m}$, $5 \mathrm{H}), 6.78(\mathrm{~s}, 1 \mathrm{H}), 4.21(\mathrm{~d}, J=17.1 \mathrm{~Hz}, \mathrm{AB}$ system, $1 \mathrm{H}), 3.81-3.47(\mathrm{~m}, 4 \mathrm{H}), 2.70-2.63(\mathrm{~m}, 1 \mathrm{H}), 2.56-2.42$ (m, 1H), 2.06-1.95 (m, 1H), 1.24-0.81 (m, 9H); ${ }^{13} \mathrm{C}-\mathrm{NMR}\left(75 \mathrm{MHz}, \mathrm{CDCl}_{3}\right) \delta 148.6,138.0,132.5,129.1$, 128.4, 127.6, 124.8, 124.6, 61.0, 59.1, 41.4, 31.7, 28.3, 20.1 (2C), 9.6, 8.9; IR (KBr) 3002, 2960, 2925, 2870, $1668,1454,1359,1344,1246,1090 v_{\max } / \mathrm{cm}^{-1}$; m.p. $107-108{ }^{\circ} \mathrm{C}$; MS (ESI) $\mathrm{m} / z$ Calcd for $\mathrm{C}_{19} \mathrm{H}_{25} \mathrm{~N}_{4}{ }^{+}$: 309.2074; found: $309.2072[\mathrm{M}+\mathrm{H}]^{+}$.

(E)- $N$-(7-benzyl-6-isopropyl-7,8-dihydroimidazo[1,5-a]pyrazin-5(6H)-ylidene)-2-phenylethanamine (51). The crude material was purified by column chromatography (Pet/EtOAc 90:10 and Pet/EtOAc 70:30) to give the product as brown oil (113.5 mg, yield 61\%). ${ }^{1} \mathrm{H}-\mathrm{NMR}\left(300 \mathrm{MHz}, \mathrm{CDCl}_{3}\right) \delta 8.34(\mathrm{~s}$, $1 \mathrm{H}), 7.29-7.16(\mathrm{~m}, 10 \mathrm{H}), 6.76(\mathrm{~s}, 1 \mathrm{H}), 4.12(\mathrm{~d}, J=17.1 \mathrm{~Hz}, \mathrm{AB}$ system, $1 \mathrm{H}), 3.69(\mathrm{~d}, J=17.1 \mathrm{~Hz}, \mathrm{AB}$ system, $1 \mathrm{H}), 3.54-3.47(\mathrm{~m}, 3 \mathrm{H}), 3.34-3.20(\mathrm{~m}, 2 \mathrm{H}), 3.05-2.84(\mathrm{~m}, 2 \mathrm{H}), 2.05-1.90(\mathrm{~m}, 1 \mathrm{H}), 1.14-1.11(\mathrm{~m}$, $3 \mathrm{H}), 0.86-0.83(\mathrm{~m}, 3 \mathrm{H}) ;{ }^{13} \mathrm{C}-\mathrm{NMR}\left(75 \mathrm{MHz}, \mathrm{CDCl}_{3}\right) \delta 149.6,140.0,138.1,132.8,129.2,128.6$ (2C), 128.5, 127.6, 126.4, 125.2, 124.8, 61.3, 58.6, 51.5, 40.8, 37.8, 28.4, 20.1, 19.9; IR (neat) 2960, 1672, 1494, 1456, 1353, $1218,1103,743,698 v_{\max } / \mathrm{cm}^{-1}$; MS (ESI) $\mathrm{m} / z$ Calcd for $\mathrm{C}_{24} \mathrm{H}_{29} \mathrm{~N}_{4}{ }^{+}$: 373.2387; found: fragment ion (loss of $\mathrm{N}$-methylene-2-phenylethanamine moiety): found: $242.1654[\mathrm{M}+\mathrm{H}]^{+}$.

(E)-methyl2-((7-benzyl-6-isopropyl-7,8-dihydroimidazo[1,5-a]pyrazin-5(6H)-ylidene)amino)-acetate (52). The crude material was purified by column chromatography (Pet/EtOAc 70:30) to give the product as brown oil (92 mg, yield 54\%). ${ }^{1} \mathrm{H}-\mathrm{NMR}\left(300 \mathrm{MHz}, \mathrm{CDCl}_{3}\right) \delta 8.33(\mathrm{~s}, 1 \mathrm{H}), 7.28-7.25(\mathrm{~m}, 5 \mathrm{H})$, $6.77(\mathrm{~s}, 1 \mathrm{H}), 4.21(\mathrm{~d}, J=17.4 \mathrm{~Hz}, \mathrm{AB}$ system, $1 \mathrm{H}), 4.05(\mathrm{br} \mathrm{s}, 2 \mathrm{H}), 3.81-3.76(\mathrm{~m}, 5 \mathrm{H}), 3.60(\mathrm{~d}, J=13.2 \mathrm{~Hz}$, AB system, $1 \mathrm{H}), 3.27(\mathrm{~d}, J=10.7 \mathrm{~Hz}, 1 \mathrm{H}), 2.02-1.93(\mathrm{~m}, 1 \mathrm{H}) 1.13(\mathrm{~d}, J=6.4 \mathrm{~Hz}, 3 \mathrm{H}), 0.86(\mathrm{~d}, J=7.8 \mathrm{~Hz}$, $3 \mathrm{H}) ;{ }^{13} \mathrm{C}-\mathrm{NMR}\left(75 \mathrm{MHz}, \mathrm{CDCl}_{3}\right) \delta 170.2,152.9,137.7,133.2,128.8,128.6,127.8,125.0(2 \mathrm{C}), 61.0,59.0$, 52.3, 51.2, 41.2, 28.3, 20.0, 19.8; IR (neat) 2961, 1743, 1678, 1465, 1358, 1198, 1178, $741 \mathrm{v}_{\max } / \mathrm{cm}^{-1}$; MS (ESI) $\mathrm{m} / \mathrm{z}$ Calcd for $\mathrm{C}_{19} \mathrm{H}_{25} \mathrm{~N}_{4} \mathrm{O}_{2}{ }^{+}$: 341.1973 ; found: $341.1971[\mathrm{M}+\mathrm{H}]^{+}$.

(E)-N-(7-benzyl-6-hexyl-7,8-dihydroimidazo[1,2-a]pyrazin-5(6H)-ylidene)pentan-1-amine

(53).

The crude material was purified by column chromatography (Pet/EtOAc 80:20) to give the product as yellow oil (129 mg, yield 68\%). ${ }^{1} \mathrm{H}-\mathrm{NMR}\left(300 \mathrm{MHz}, \mathrm{CDCl}_{3}\right) \delta 7.62(\mathrm{~s}, 1 \mathrm{H}), 7.31-7.29(\mathrm{~m}, 5 \mathrm{H}), 7.00(\mathrm{~s}$, $1 \mathrm{H}), 4.25(\mathrm{~d}, J=17.8 \mathrm{~Hz}, \mathrm{AB}$ system, $1 \mathrm{H}), 3.93(\mathrm{~d}, J=17.8 \mathrm{~Hz}, \mathrm{AB}$ system, $1 \mathrm{H}), 3.89-3.76(\mathrm{~m}, 3 \mathrm{H}), 3.58$ $(\mathrm{d}, J=13.2 \mathrm{~Hz}, \mathrm{AB}$ system, $1 \mathrm{H}), 3.23(\mathrm{t}, J=6.7 \mathrm{~Hz}, 2 \mathrm{H}) 1.75-1.27(\mathrm{~m}, 15 \mathrm{H}), 0.94-0.87(\mathrm{~m}, 6 \mathrm{H}) ;{ }^{13} \mathrm{C}-\mathrm{NMR}$ $\left(75 \mathrm{MHz}, \mathrm{CDCl}_{3}\right) \delta 150.9,143.6,137.7,129.0,128.6,128.4,127.8,113.5,59.4,54.4,48.7,44.5,31.8,31.0$, 29.7, 28.8 (2C), 26.0, 22.7, 22.6, 14.2 (2C); IR (neat) 2927, 2857, 1670, 1491, 1455, 1413, 1289, 1093, 889, 743, $698 v_{\max } / \mathrm{cm}^{-1}$; MS (ESI) $\mathrm{m} / z$ Calcd for $\mathrm{C}_{24} \mathrm{H}_{37} \mathrm{~N}_{4}{ }^{+}$: 381.3013; found: $381.3006[\mathrm{M}+\mathrm{H}]^{+}$.

(E)-N-(7-benzyl-6-isopropyl-7,8-dihydroimidazo[1,2-a]pyrazin-5(6H)-ylidene)-1-phenylmethanamine (54). The crude material was purified by column chromatography (Pet/EtOAc 80:20) to give the product as yellow oil (147 mg, yield 77\%). ${ }^{1} \mathrm{H}-\mathrm{NMR}\left(300 \mathrm{MHz}, \mathrm{CDCl}_{3}\right) \delta 7.69(\mathrm{~s}, 1 \mathrm{H}), 7.36-7.23(\mathrm{~m}$, $10 \mathrm{H}), 7.03(\mathrm{~s}, 1 \mathrm{H}), 4.54-4.33(\mathrm{~m}, 3 \mathrm{H}), 3.96-3.80(\mathrm{~m}, 2 \mathrm{H}), 3.61-3.51(\mathrm{~m}, 2 \mathrm{H}), 2.06-1.96(\mathrm{~m}, 1 \mathrm{H}), 1.19(\mathrm{~d}$, $J=6.4 \mathrm{~Hz}, 3 \mathrm{H}), 0.93(\mathrm{~d}, J=6.4 \mathrm{~Hz}, 3 \mathrm{H}) ;{ }^{13} \mathrm{C}-\mathrm{NMR}\left(75 \mathrm{MHz}, \mathrm{CDCl}_{3}\right) \delta 150.8,143.9,139.6,137.7,128.9$, 128.6, 128.5 (2C), 127.8, 127.5, 127.0, 114.0, 60.7, 59.6, 53.1, 45.0, 28.6, 20.2, 20.0; IR (neat) 2961, 1671, 1491, 1410, 1289, 1258, 1100, 737, $697 v_{\max } / \mathrm{cm}^{-1}$; MS (ESI) $\mathrm{m} / z$ Calcd for $\mathrm{C}_{23} \mathrm{H}_{27} \mathrm{~N}_{4}{ }^{+}$: 359.2231; found: $359.2229[\mathrm{M}+\mathrm{H}]^{+}$. 
(E)-N-(7-benzyl-6-isopropyl-7,8-dihydroimidazo[1,2-a]pyrazin-5(6H)-ylidene)cyclopropanamine (55). The crude material was purified by column chromatography (Pet/EtOAc 70:30) to give the product as yellow oil (91 mg, yield 56\%). ${ }^{1} \mathrm{H}-\mathrm{NMR}\left(300 \mathrm{MHz}, \mathrm{CDCl}_{3}\right) \delta 7.45(\mathrm{br} \mathrm{d}, 1 \mathrm{H}), 7.29-7.24(\mathrm{~m}$, $5 \mathrm{H}), 6.96(\mathrm{br} \mathrm{d}, 1 \mathrm{H}), 4.30(\mathrm{~d}, J=18.0 \mathrm{~Hz}, \mathrm{AB}$ system, $1 \mathrm{H}), 3.92-3.78(\mathrm{~m}, 2 \mathrm{H}), 3.61-3.47(\mathrm{~m}, 2 \mathrm{H}), 2.65-2.61$ (m, 1H), 2.02-1.86 (m, 1H), 1.19-0.78 (m, 10H); ${ }^{13} \mathrm{C}-\mathrm{NMR}\left(75 \mathrm{MHz}, \mathrm{CDCl}_{3}\right) \delta 149.2,143.3,137.7,129.4$, $129.1,128.4,128.2,127.6,113.6,60.5,59.6,45.1,31.9,28.3,20.1,20.0,9.6,8.9$; IR (neat) 2962, 1665, 1530, $1488,1415,1367,1289,1261,1087,743,698 v_{\max } / \mathrm{cm}^{-1}$; MS (ESI) $\mathrm{m} / \mathrm{z}$ Calcd for $\mathrm{C}_{19} \mathrm{H}_{25} \mathrm{~N}_{4}{ }^{+}$: 309.2074; found: $309.2072[\mathrm{M}+\mathrm{H}]^{+}$.

(E)-N-(7-benzyl-6-phenyl-7,8-dihydroimidazo[1,2-a]pyrazin-5(6H)-ylidene)cyclohexanamine (56). The crude material was purified by column chromatography (Pet/EtOAc 90:10 and Pet/EtOAc 70:30) to give the product as yellow oil (114 mg, yield 56\%). ${ }^{1} \mathrm{H}-\mathrm{NMR}\left(300 \mathrm{MHz}, \mathrm{CDCl}_{3}\right) \delta 7.78(\mathrm{br} \mathrm{d}, 1 \mathrm{H})$, 7.41-7.23 (m, 10H), $7.02(\mathrm{br} \mathrm{d}, 1 \mathrm{H}), 5.04(\mathrm{~s}, 1 \mathrm{H}), 3.91-3.67(\mathrm{~m}, 4 \mathrm{H}), 3.09-2.99(\mathrm{~m}, 1 \mathrm{H}), 1.73-1.00(\mathrm{~m}, 10 \mathrm{H})$; ${ }^{13} \mathrm{C}-\mathrm{NMR}\left(75 \mathrm{MHz}, \mathrm{CDCl}_{3}\right) \delta 146.3,144.3,137.5,134.7,129.0,128.9,128.7,128.6,128.5,128.1,127.9$, 113.7, 58.9, 58.7, 57.2, 44.9, 34.3, 33.6, 25.5, 24.2, 24.1; IR (neat) 2928, 2853, 1668, 1536, 1492, 1450, 1412, 1289, 1090, 740, $698 v_{\max } / \mathrm{cm}^{-1}$; MS (ESI) $\mathrm{m} / z$ Calcd for $\mathrm{C}_{25} \mathrm{H}_{29} \mathrm{~N}_{4}{ }^{+}: 385.2387$; found: $385.2387[\mathrm{M}+\mathrm{H}]^{+}$.

(E)-N-(7-benzyl-6-isopropyl-7,8-dihydroimidazo[1,2-a]pyrazin-5(6H)-ylidene)-[1,1'-biphenyl]-4-amine

(57). The crude material was purified by column chromatography (Pet/EtOAc 90:10 and Pet/EtOAc 70:30) to give the product as yellow oil (158 mg, yield 70\%). ${ }^{1} \mathrm{H}-\mathrm{NMR}\left(300 \mathrm{MHz}, \mathrm{CDCl}_{3}\right) \delta 7.77(\mathrm{br} \mathrm{d}$, $1 \mathrm{H}), 7.77-7.29(\mathrm{~m}, 12 \mathrm{H}), 7.11(\mathrm{br} \mathrm{d}, 1 \mathrm{H}), 6.86(\mathrm{br} \mathrm{d}, 2 \mathrm{H}), 4.32-4.14(\mathrm{~m}, 1 \mathrm{H}), 4.02-3.84(\mathrm{~m}, 3 \mathrm{H}), 3.70-3.63$ (m, 1H), 2.07-1.97 (m, 1H), 1.11 (br d, 3H), $0.82\left(\right.$ br s 3H); ${ }^{13} \mathrm{C}-\mathrm{NMR}\left(75 \mathrm{MHz}, \mathrm{CDCl}_{3}\right) \delta 150.9,146.0$, 144.7, 140.6, 137.8, 137.0, 129.3, 128.9, 128.7, 128.6, 127.8, 127.7, 127.2, 126.8, 120.8, 114.1, 62.8, 59.7, 44.1, 28.9, 20.4 (2C); IR (neat) 2963, 1667, 1535, 1484, 1407, 1259, 1220, 908, 848, 730, $697 v_{\max } / \mathrm{cm}^{-1}$; MS (ESI) $m / z$ Calcd for $\mathrm{C}_{28} \mathrm{H}_{29} \mathrm{~N}_{4}^{+}$: 421.2387; found: $421.2386[\mathrm{M}+\mathrm{H}]^{+}$.

(E)-N-(7-benzyl-6-(pyridin-3-yl)-7,8-dihydroimidazo[1,2-a]pyrazin-5(6H)-ylidene)-2-methylpropan2-amine (58). The crude material was purified by column chromatography (Pet/EtOAc 50:50 and EtOAc) to give the product as yellow oil $(86 \mathrm{mg}$, yield $45 \%) .{ }^{1} \mathrm{H}-\mathrm{NMR}\left(300 \mathrm{MHz}, \mathrm{CDCl}_{3}\right) \delta 8.56(\mathrm{br} \mathrm{d}$, $2 \mathrm{H}), 7.76(\mathrm{~s}, 1 \mathrm{H}), 7.46-7.22(\mathrm{~m}, 7 \mathrm{H}), 7.00(\mathrm{~s}, 1 \mathrm{H}), 5.19(\mathrm{~s}, 1 \mathrm{H}), 3.91-3.81(\mathrm{~m}, 3 \mathrm{H}), 3.64(\mathrm{~d}, J=18.3 \mathrm{~Hz}, \mathrm{AB}$ system, $1 \mathrm{H}), 1.12(\mathrm{~s}, 9 \mathrm{H}) ;{ }^{13} \mathrm{C}-\mathrm{NMR}\left(75 \mathrm{MHz}, \mathrm{CDCl}_{3}\right) \delta 157.8,150.3,149.8,143.8,142.5,137.2,135.7$, 131.4, 128.8, 128.7, 128.0, 123.5, 114.1, 59.5, 58.5, 55.0, 44.7, 31.3; IR (neat) 2970, 1674, 1454, 1418, 1360, $1294,1206,1092,911,729,700 v_{\max } / \mathrm{cm}^{-1}$; MS (ESI) $\mathrm{m} / \mathrm{z}$ Calcd for $\mathrm{C}_{22} \mathrm{H}_{26} \mathrm{~N}_{5}{ }^{+}: 360.2183$; found: 360.2181 $[\mathrm{M}+\mathrm{H}]^{+}$.

(E)-N-(7-benzyl-6-(furan-2-yl)-7,8-dihydroimidazo[1,2-a]pyrazin-5(6H)-ylidene)-2-methylpropan2-amine (59). The crude material was purified by column chromatography (Pet/EtOAc 90:10 and Pet/EtOAc 70:30) to give the product as yellow oil (93 mg, yield 50\%). ${ }^{1} \mathrm{H}-\mathrm{NMR}\left(300 \mathrm{MHz}, \mathrm{CDCl}_{3}\right)$ $\delta 7.63(\mathrm{br} \mathrm{d}, 1 \mathrm{H}), 7.45-7.26(\mathrm{~m}, 6 \mathrm{H}), 6.98(\mathrm{br} \mathrm{d}, 1 \mathrm{H}), 6.31(\mathrm{br} \mathrm{d}, 1 \mathrm{H}), 5.94-5.93(\mathrm{~m}, 1 \mathrm{H}), 5.19(\mathrm{~m}, 1 \mathrm{H})$, 3.99-3.57 (m, 4H), $1.06(\mathrm{~s}, 9 \mathrm{H}) ;{ }^{13} \mathrm{C}-\mathrm{NMR}\left(75 \mathrm{MHz}, \mathrm{CDCl}_{3}\right) \delta$ 148.0, 144.7, 143.1, 142.7, 137.2, 129.3, $128.7,128.2,127.9,114.2,110.6,110.5,58.4,54.9,54.5,46.7,30.9$; IR (neat) 2970, 1684, 1542, 1493, 1418 , 1364, 1208, 1087, 1014, 742, $699 v_{\max } / \mathrm{cm}^{-1}$; MS (ESI) $\mathrm{m} / z$ Calcd for $\mathrm{C}_{21} \mathrm{H}_{25} \mathrm{~N}_{4} \mathrm{O}^{+}$: 349.2023; found: $349.2022[\mathrm{M}+\mathrm{H}]^{+}$.

(E)-N-(7-(4-chlorobenzyl)-6-phenyl-7,8-dihydroimidazo[1,5-a]pyrazin-5(6H)-ylidene)cyclohexanamine (60). The crude material was purified by column chromatography (Pet/EtOAc 70:30) to give the product as orange oil $(167 \mathrm{mg}$, yield $80 \%) .{ }^{1} \mathrm{H}-\mathrm{NMR}\left(300 \mathrm{MHz}, \mathrm{CDCl}_{3}\right) \delta 8.48(\mathrm{~s}, 1 \mathrm{H}), 7.41-7.26(\mathrm{~m}, 9 \mathrm{H})$, $6.74(\mathrm{~s}, 1 \mathrm{H}), 4.99(\mathrm{~s}, 1 \mathrm{H}), 3.81-3.63(\mathrm{~m}, 4 \mathrm{H}), 3.12-3.08(\mathrm{~m}, 1 \mathrm{H}), 1.74-0.88(\mathrm{~m}, 10 \mathrm{H}) ;{ }^{13} \mathrm{C}-\mathrm{NMR}(75 \mathrm{MHz}$, $\left.\mathrm{CDCl}_{3}\right) \delta 145.3,136.3,134.7,133.6,133.0,130.2,128.9(2 \mathrm{C}), 128.6,128.2,125.1,125.0,59.8,57.6,56.9$, 41.1, 34.3, 33.5, 25.5, 24.2, 24.1; IR (neat) 2928, 2854, 1669, 1488, 1462, 1347, 1191, 1088, 803, 733, 699, $653 v_{\max } / \mathrm{cm}^{-1}$; MS (ESI) $\mathrm{m} / \mathrm{z}$ Calcd for $\mathrm{C}_{25} \mathrm{H}_{28} \mathrm{ClN}_{4}{ }^{+}$: 419.1997; found: $419.1998[\mathrm{M}+\mathrm{H}]^{+}$.

(E)-1-((3r,5r,7r)-adamantan-1-yl)-N-(7-(4-chlorobenzyl)-6-phenyl-7,8-dihydroimidazo[1,5- $a$ ]pyrazin$5(6 H)$-ylidene)methanamine (61). The crude material was purified by column chromatography 
(Pet/EtOAc 90:10 and Pet/EtOAc 70:30) to give the product as yellow oil (193 mg, yield 82\%). ${ }^{1} \mathrm{H}-\mathrm{NMR}$ $\left(300 \mathrm{MHz}, \mathrm{CDCl}_{3}\right) \delta 8.49(\mathrm{~s}, 1 \mathrm{H}), 7.36-7.26(\mathrm{~m}, 9 \mathrm{H}), 6.71(\mathrm{~s}, 1 \mathrm{H}), 5.18(\mathrm{~s}, 1 \mathrm{H}), 3.81(\mathrm{~s}, 2 \mathrm{H}), 3.59(\mathrm{~s}$, 2H), 1.96 (s, 3H), 1.70-1.50 (m, 12H); ${ }^{13} \mathrm{C}-\mathrm{NMR}\left(75 \mathrm{MHz}, \mathrm{CDCl}_{3}\right) \delta 142.4,136.5,135.9,133.6,133.3$, 130.2, 128.9, 128.8, 128.6, 128.4, 125.6, 124.9, 63.0, 57.5, 56.1, 44.0, 41.0, 36.3, 29.7; IR (neat) 2906, 2850, $1675,1491,1459,1340,1190,1088,909,804,729,698 v_{\max } / \mathrm{cm}^{-1}$; MS (ESI) $\mathrm{m} / z$ Calcd for $\mathrm{C}_{29} \mathrm{H}_{32} \mathrm{ClN}_{4}{ }^{+}$: 471.2310; found: $471.2320[\mathrm{M}+\mathrm{H}]^{+}$.

(E)-methyl2-((7-(4-chlorobenzyl)-6-hexyl-7,8-dihydroimidazo[1,5-a]pyrazin-5(6H)-ylidene)amino) acetate (62). The crude material was purified by column chromatography (Pet/EtOAc 80:20 and Pet/EtOAc 60:40) to give the product as yellow oil (156 mg, yield $75 \%) .{ }^{1} \mathrm{H}-\mathrm{NMR}\left(300 \mathrm{MHz}, \mathrm{CDCl}_{3}\right) \delta$ $8.35(\mathrm{~s}, 1 \mathrm{H}), 7.297 .18(\mathrm{~m}, 4 \mathrm{H}), 6.78(\mathrm{~s}, 1 \mathrm{H}), 4.16-4.12(\mathrm{~m}, 3 \mathrm{H}), 3.78-3.52(\mathrm{~m}, 7 \mathrm{H}), 1.76-1.16(\mathrm{~m}, 10 \mathrm{H})$, $0.87-0.85(\mathrm{~m}, 3 \mathrm{H}) ;{ }^{13} \mathrm{C}-\mathrm{NMR}\left(75 \mathrm{MHz}, \mathrm{CDCl}_{3}\right) \delta 170.0,154.0,136.3,133.6,133.4,130.2,128.8,125.4$, 124.3, 58.2, 55.5, 52.3, 50.2, 40.5, 31.7, 29.0, 28.8, 26.0, 22.6, 14.1; IR (neat) 2927, 2856, 1746, 1673, 1464, $1351,1197,1176,1087,1014,807,652 v_{\max } / \mathrm{cm}^{-1}$; MS (ESI) $\mathrm{m} / z$ Calcd for $\mathrm{C}_{22} \mathrm{H}_{30} \mathrm{ClN}_{4} \mathrm{O}_{2}{ }^{+}$: 417.2052; found: $417.2051[\mathrm{M}+\mathrm{H}]^{+}$.

(E)-N-(7-(4-chlorobenzyl)-6-(furan-2-yl)-7,8-dihydroimidazo[1,5- $a$ ]pyrazin-5(6H)-ylidene)-2-

methylpropan-2-amine (63). The crude material was purified by column chromatography (Pet/EtOAc 90:10 and Pet/EtOAc 70:30) to give the product as yellow oil (88 mg, yield 46\%). ${ }^{1} \mathrm{H}-\mathrm{NMR}$ $\left(300 \mathrm{MHz}, \mathrm{CDCl}_{3}\right) \delta 8.33(\mathrm{~s}, 1 \mathrm{H}), 7.43-7.34(\mathrm{~m}, 5 \mathrm{H}), 6.77(\mathrm{br} \mathrm{d}, 1 \mathrm{H}), 6.33-6.32(\mathrm{~m}, 1 \mathrm{H}), 6.01(\mathrm{br} \mathrm{d}, 1 \mathrm{H})$, $5.13(\mathrm{~s}, 1 \mathrm{H}), 3.83-3.54(\mathrm{~m}, 4 \mathrm{H}) 1.10(\mathrm{~s}, 9 \mathrm{H}) ;{ }^{13} \mathrm{C}-\mathrm{NMR}\left(75 \mathrm{MHz}, \mathrm{CDCl}_{3}\right) \delta 147.8,141.7,135.8,133.7,133.1$, 130.4, 128.8, 126.0, 124.0, 110.7, 110.5, 57.6, 55.6, 54.6, 43.0, 30.8; IR (neat) 2969, 1685, 1490, 1461, 1346, 1201, 1089, 1014, 809, 740, $654 v_{\max } / \mathrm{cm}^{-1}$; MS (ESI) $\mathrm{m} / \mathrm{z}$ Calcd for $\mathrm{C}_{21} \mathrm{H}_{24} \mathrm{ClN}_{4} \mathrm{O}^{+}$: 383.1634; found: $383.1633[\mathrm{M}+\mathrm{H}]^{+}$.

\section{(E)-N-(2-benzyl-3-hexyl-2,3-dihydrobenzo[4,5]imidazo[1,2-a]pyrazin-4(1H)-ylidene)-2-}

methylpropan-2-amine (64). The crude material was purified by column chromatography Pet/EtOAc 95:5 and Pet/EtOAc 80:20) to give the product as yellow oil (208 mg, yield 78\%). ${ }^{1} \mathrm{H}-\mathrm{NMR}$ $\left(300 \mathrm{MHz}, \mathrm{CDCl}_{3}\right) \delta 8.50-8.47(\mathrm{~m}, 1 \mathrm{H}), 7.72-7.69(\mathrm{~m}, 1 \mathrm{H}), 7.33-7.26(\mathrm{~m}, 7 \mathrm{H}), 4.40(\mathrm{~d}, J=18.3 \mathrm{~Hz}, \mathrm{AB}$ system, $1 \mathrm{H}), 4.09-4.00(\mathrm{~m}, 2 \mathrm{H}), 3.85$ (br s, $2 \mathrm{H}), 1.91-1.71(\mathrm{~m}, 4 \mathrm{H}), 1.58-1.35(\mathrm{~m}, 15 \mathrm{H}), 0.92-0.94(\mathrm{~m}, 3 \mathrm{H})$; ${ }^{13} \mathrm{C}-\mathrm{NMR}\left(75 \mathrm{MHz}, \mathrm{CDCl}_{3}\right) \delta$ 149.9, 149.3, 143.2, 137.9, 132.6, 128.6 (2C), 127.7, 123.5 (2C), 118.9, 116.6, 59.3, 59.2, 55.0, 44.1, 31.8, 30.2, 28.9 (2C), 26.2, 22.7, 14.1; IR (neat) 2961, 2927, 1670, 1539, 1448, 1361, 1343, 1164, $746 v_{\max } / \mathrm{cm}^{-1}$; MS (ESI) $\mathrm{m} / z$ Calcd for $\mathrm{C}_{27} \mathrm{H}_{37} \mathrm{~N}_{4}^{+}$: 417.3013; found: $417.3010[\mathrm{M}+\mathrm{H}]^{+}$.

(E)-N-(2-benzyl-3-(4-chlorophenyl)-2,3-dihydrobenzo[4,5]imidazo[1,2-a]pyrazin-4(1H)-ylidene)

pentan-1-amine (65). The crude material was purified by column chromatography (Pet/EtOAc 95:5 and Pet/EtOAc 90:10) to give the product as yellow oil (119 mg, yield 52\%). ${ }^{1} \mathrm{H}-\mathrm{NMR}(300 \mathrm{MHz}$, $\left.\mathrm{CDCl}_{3}\right) \delta 8.64-8.62(\mathrm{~m}, 1 \mathrm{H}), 7.75-7.73(\mathrm{~m}, 1 \mathrm{H}), 7.40-7.20(\mathrm{~m}, 11 \mathrm{H}), 5.10(\mathrm{~s}, 1 \mathrm{H}), 4.02-3.95(\mathrm{~m}, 3 \mathrm{H})$, $3.77(\mathrm{~d}, J=12.8 \mathrm{~Hz}, \mathrm{AB}$ system, $1 \mathrm{H}), 3.32-3.11(\mathrm{~m}, 2 \mathrm{H}), 1.71-1.67(\mathrm{~m}, 2 \mathrm{H}), 1.41-1.30(\mathrm{~m}, 4 \mathrm{H}), 0.92(\mathrm{t}$, $J=6.9 \mathrm{~Hz}, 3 \mathrm{H}) ;{ }^{13} \mathrm{C}-\mathrm{NMR}\left(75 \mathrm{MHz}, \mathrm{CDCl}_{3}\right) \delta 150.0,149.5,143.1,137.2,134.6,132.9,132.2,129.6,129.2$, 129.1, 128.9, 128.1, 124.1 (2C), 119.3, 116.7, 58.9, 58.8, 49.0, 45.5, 30.9, 29.7, 22.5, 14.1; IR (neat) 2928, $2855,1665,1650,1489,1449,1357,1167,1090,746,698 v_{\max } / \mathrm{cm}^{-1}$; MS (ESI) $\mathrm{m} / z$ Calcd for $\mathrm{C}_{28} \mathrm{H}_{30} \mathrm{ClN}_{4}{ }^{+}$: 457.2154; found: $457.2157[\mathrm{M}+\mathrm{H}]^{+}$.

\section{(E)-N-(2-benzyl-3-hexyl-2,3-dihydrobenzo[4,5]imidazo[1,2-a]pyrazin-4(1H)-ylidene)}

cyclohexanamine (66). The crude material was purified by column chromatography (Pet/EtOAc 95:5 and Pet/EtOAc 90:10) to give the product as yellow oil (130 mg, yield 59\%). ${ }^{1} \mathrm{H}-\mathrm{NMR}(300 \mathrm{MHz}$, $\left.\mathrm{CDCl}_{3}\right) \delta 8.55-8.52(\mathrm{~m}, 1 \mathrm{H}), 7.73-7.70(\mathrm{~m}, 1 \mathrm{H}), 7.35-7.26(\mathrm{~m}, 7 \mathrm{H}), 4.43(\mathrm{~d}, J=18.1 \mathrm{~Hz}, \mathrm{AB}$ system, $1 \mathrm{H})$, $4.11(\mathrm{~d}, J=18.1 \mathrm{~Hz}, \mathrm{AB}$ system, $1 \mathrm{H}), 3.98\left(\mathrm{dd}, J_{1}=10.7 \mathrm{~Hz}, J_{2}=4.3 \mathrm{~Hz}, 1 \mathrm{H}\right), 3.83(\mathrm{~d}, J=13.1 \mathrm{~Hz}$, AB system, $1 \mathrm{H}), 3.70(\mathrm{~d}, J=13.1 \mathrm{~Hz}, \mathrm{AB}$ system, $1 \mathrm{H}), 3.38-3.30(\mathrm{~m}, 1 \mathrm{H}), 1.93-1.29(\mathrm{~m}, 20 \mathrm{H}), 0.89(\mathrm{t}$, $J=6.4 \mathrm{~Hz}, 3 \mathrm{H}) ;{ }^{13} \mathrm{C}-\mathrm{NMR}\left(75 \mathrm{MHz}, \mathrm{CDCl}_{3}\right) \delta 150.8,149.5,143.1,137.7,132.4,128.9,128.5,127.8,123.7$ (2C), 119.0, 116.8, 59.5, 56.9, 55.1, 44.8, 34.9, 34.7, 31.7, 29.8, 28.7, 25.8 (2C), 24.3, 24.2, 22.6, 14.1; IR 
(neat) 2926, 2853, 1666, 1540, 1449, 1346, 1173, 744, $698 v_{\max } / \mathrm{cm}^{-1}$; MS (ESI) $\mathrm{m} / z$ Calcd for $\mathrm{C}_{29} \mathrm{H}_{39} \mathrm{~N}_{4}{ }^{+}$: 443.3170; found: $443.3170[\mathrm{M}+\mathrm{H}]^{+}$.

(E)-methyl2-((3-hexyl-2-phenethyl-2,3-dihydrobenzo[4,5]imidazo[1,2-a]pyrazin-4(1H)-ylidene) amino)acetate (67). The crude material was purified by column chromatography Pet/EtOAc 95:5 and Pet/EtOAc 90:10) to give the product as yellow oil (145 mg, yield $65 \%) .{ }^{1} \mathrm{H}-\mathrm{NMR}\left(300 \mathrm{MHz}, \mathrm{CDCl}_{3}\right) \delta$ 8.53-8.50 (m, 1H), 7.70-7.67 (m, 1H), 7.34-7.15 (m, 7H), $4.38(\mathrm{~d}, J=18.3 \mathrm{~Hz}, \mathrm{AB}$ system, $1 \mathrm{H}), 4.33(\mathrm{br}$ $\mathrm{d}, 1 \mathrm{H}), 4.15(\mathrm{~d}, J=18.0 \mathrm{~Hz}, \mathrm{AB}$ system, $1 \mathrm{H}), 3.90-3.77(\mathrm{~m}, 5 \mathrm{H}), 2.93-2.78(\mathrm{~m}, 4 \mathrm{H}), 1.84-1.51(\mathrm{~m}, 3 \mathrm{H})$, 1.37-1.23 (m, 7H), 0.91-0.87 (m, 3H); ${ }^{13} \mathrm{C}-\mathrm{NMR}\left(75 \mathrm{MHz}, \mathrm{CDCl}_{3}\right) \delta 170.5,156.7,149.3,142.9,139.6$, 132.2, 128.9, 128.6, 126.5, 124.5, 124.3, 119.1, 116.9, 57.5, 57.1, 52.4, 50.8, 44.6, 35.2, 31.8, 29.4, 29.0, 26.2, 22.7, 14.1; IR (neat) 2928, 2885, 1746, 1670, 1451, 1375, 1200, 1174, 746, $699 v_{\max } / \mathrm{cm}^{-1}$; MS (ESI) $\mathrm{m} / \mathrm{z}$ Calcd for $\mathrm{C}_{27} \mathrm{H}_{35} \mathrm{~N}_{4} \mathrm{O}_{2}{ }^{+}$: 447.2755; found: $447.2757[\mathrm{M}+\mathrm{H}]^{+}$.

(E)-N-(7-hexyl-6-(4-methoxyphenyl)-7,8-dihydroimidazo[1,5-a]pyrazin-5(6H)-ylidene)-2-

methylpropan-2-amine (68). The crude material was purified by column chromatography ( $n$-hexane/ EtOAc 97:3) to give the product as brownish solid (162 mg, 85\% yield). ${ }^{1} \mathrm{H}-\mathrm{NMR}(400 \mathrm{MHz}$, $\left.\mathrm{CDCl}_{3}\right) \delta 8.34(\mathrm{~s}, 1 \mathrm{H}), 7.08(\mathrm{~d}, J=8.4 \mathrm{~Hz}, 2 \mathrm{H}), 6.77(\mathrm{~d}, J=8.4 \mathrm{~Hz}, 2 \mathrm{H}), 6.65(\mathrm{~s}, 1 \mathrm{H}), 5.03(\mathrm{~s}, 1 \mathrm{H}), 3.71(\mathrm{~s}$, $3 \mathrm{H}), 3.52\left(\mathrm{dd}, J_{a b}=16.4 \mathrm{~Hz}, 2 \mathrm{H}\right), 2.62-2.48(\mathrm{~m}, 2 \mathrm{H}), 1.56-1.49(\mathrm{~m}, 2 \mathrm{H}), 1.35-1.26(\mathrm{~m}, 6 \mathrm{H}), 1.20(\mathrm{~s}, 9 \mathrm{H})$, $0.86-0.83(\mathrm{~m}, 3 \mathrm{H}) ;{ }^{13} \mathrm{C}-\mathrm{NMR}\left(100 \mathrm{MHz}, \mathrm{CDCl}_{3}\right) \delta 159.2,144.2,132.9,129.7,127.5,125.8,124.5,113.8$, 62.7, 55.1, 54.5, 53.6, 40.5, 31.6, 31.3, 28.1, 26.8, 22.5, 14.0. IR (neat) 2965, 2929, 2861, 2833, 1668, 1511, 1460, 1338, 1246, 1037, $819 v_{\max } / \mathrm{cm}^{-1}$; Mp 78-79 ${ }^{\circ} \mathrm{C}$; MS (ESI) $\mathrm{m} / z$ Calcd for $\mathrm{C}_{23} \mathrm{H}_{35} \mathrm{~N}_{4} \mathrm{O}^{+}$: 383.2806 ; found: fragment ion (loss of 2-methyl-N-methylenepropan-2-amine moiety): $300.2072[\mathrm{M}+\mathrm{H}]^{+}$.

(E)-4-(5-(tert-butylimino)-7-hexyl-5,6,7,8-tetrahydroimidazo[1,5-a]pyrazin-6-yl)- $N, N$-dimethylaniline (69). The crude material was purified by column chromatography ( $n$-hexane/ EtOAc 85:15) to give the product as yellowish solid (49 mg, $25 \%$ yield). ${ }^{1} \mathrm{H}-\mathrm{NMR}\left(400 \mathrm{MHz}, \mathrm{CDCl}_{3}\right) \delta 8.36(\mathrm{~s}, 1 \mathrm{H}), 7.01(\mathrm{~d}$, $J=8.0 \mathrm{~Hz}, 2 \mathrm{H}), 6.68(\mathrm{~s}, 1 \mathrm{H}), 6.60(\mathrm{~d}, J=8.0 \mathrm{~Hz}, 2 \mathrm{H}), 5.03(\mathrm{~s}, 1 \mathrm{H}), 3.57\left(\mathrm{dd}, J_{a b}=15.2 \mathrm{~Hz}, 2 \mathrm{H}\right), 2.91(\mathrm{~s}$, $6 \mathrm{H}), 2.62-2.49(\mathrm{~m}, 2 \mathrm{H}), 1.58-1.52(\mathrm{~m}, 2 \mathrm{H}), 1.39-1.30(\mathrm{~m}, 6 \mathrm{H}), 1.24(\mathrm{~s}, 9 \mathrm{H}), 0.90-0.87(\mathrm{~m}, 3 \mathrm{H}) ;{ }^{13} \mathrm{C}-\mathrm{NMR}$ $\left(100 \mathrm{MHz}, \mathrm{CDCl}_{3}\right) \delta 150.0,144.7,132.9,129.4,126.1,124.4,122.5,112.0,62.8,54.4,53.6,40.7,40.3,31.7$, 31.3, 29.7, 28.2, 26.8, 22.6, 14.0. IR (neat) 3115, 2952, 2926, 2857, 1669, 1529, 1466, 1349, 1206, 1192, 811 $v_{\max } / \mathrm{cm}^{-1}$; Mp 104-105 ${ }^{\circ} \mathrm{C}$; MS (ESI) $\mathrm{m} / z$ Calcd for $\mathrm{C}_{24} \mathrm{H}_{38} \mathrm{~N}_{5}{ }^{+}: 396.3122$; found: fragment ion (loss of 2-methyl-N-methylenepropan-2-amine moiety): $313.2388[\mathrm{M}+\mathrm{H}]^{+}$.

(E)-N-(7-hexyl-6-(naphthalen-2-yl)-7,8-dihydroimidazo[1,5-a]pyrazin-5(6H)-ylidene)-2-

methylpropan-2-amine (70). The crude material was purified by column chromatography ( $n$-hexane/ EtOAc 90:10) to give the product as yellowish solid (32 mg, 16\% yield). ${ }^{1} \mathrm{H}-\mathrm{NMR}$ (400 $\left.\mathrm{MHz}, \mathrm{CDCl}_{3}\right) \delta 8.48(\mathrm{~s}, 1 \mathrm{H}), 7.83-7.72(\mathrm{~m}, 3 \mathrm{H}), 7.54(\mathrm{~s}, 1 \mathrm{H}), 7.50-7.44(\mathrm{~m}, 3 \mathrm{H}), 6.70(\mathrm{~s}, 1 \mathrm{H}), 5.27(\mathrm{~s}$, $1 \mathrm{H}), 3.58\left(\mathrm{dd}, J_{a b}=16.4 \mathrm{~Hz}, 2 \mathrm{H}\right), 2.72-2.61(\mathrm{~m}, 2 \mathrm{H}), 1.66-1.59(\mathrm{~m}, 2 \mathrm{H}), 1.42-1.29(\mathrm{~m}, 6 \mathrm{H}), 1.26(\mathrm{~s}, 9 \mathrm{H})$, 0.93-0.90 (m, 3H); ${ }^{13} \mathrm{C}-\mathrm{NMR}\left(100 \mathrm{MHz}, \mathrm{CDCl}_{3}\right) \delta 143.9,140.4,133.6,133.0,132.9,128.5,128.1,127.5$, $127.2,126.5,126.4,126.3,125.7,124.7,63.5,54.7,53.9,40.8,31.7,31.3,28.3,26.8,22.6,14.1$. IR (neat) 3120, 3055, 2964, 2930, 2857, 1669, 1464, 1347, 1362, 1203, $795 v_{\max } / \mathrm{cm}^{-1}$; Mp 108-109 ${ }^{\circ} \mathrm{C}$; MS (ESI) $\mathrm{m} / z$ Calcd for $\mathrm{C}_{26} \mathrm{H}_{35} \mathrm{~N}_{4}{ }^{+}$: 403.2857; found: fragment ion (loss of 2-methyl-N-methylenepropan-2-amine moiety): $320.2123[\mathrm{M}+\mathrm{H}]^{+}$.

(E)-N-(6-(4-chlorophenyl)-7-hexyl-7,8-dihydroimidazo[1,5-a]pyrazin-5(6H)-ylidene)-4methoxyaniline (71). The crude material was purified by column chromatography ( $n$-hexane/ EtOAc 85:15) to give the product as colorless to yellowish oil $\left(72 \mathrm{mg}, 33 \%\right.$ yield). ${ }^{1} \mathrm{H}-\mathrm{NMR}\left(400 \mathrm{MHz}, \mathrm{CDCl}_{3}\right)$ $\delta 8.49(\mathrm{~s}, 1 \mathrm{H}), 7.30(\mathrm{~d}, J=8.4 \mathrm{~Hz}, 2 \mathrm{H}), 7.13(\mathrm{~d}, J=8.4 \mathrm{~Hz}, 2 \mathrm{H}), 6.82(\mathrm{~s}, 1 \mathrm{H}), 6.77-6.74(\mathrm{~m}, 2 \mathrm{H}), 6.60-6.58$ $(\mathrm{m}, 2 \mathrm{H}), 4.77(\mathrm{~s}, 1 \mathrm{H}), 3.76(\mathrm{~s}, 3 \mathrm{H}), 3.66\left(\mathrm{dd}, J_{a b}=16.8 \mathrm{~Hz}, 2 \mathrm{H}\right), 2.62-2.42(\mathrm{~m}, 2 \mathrm{H}), 1.46-1.39(\mathrm{~m}, 2 \mathrm{H})$, 1.32-1.21 (m, 6H), 0.88-0.85 (m, 3H); ${ }^{13} \mathrm{C}-\mathrm{NMR}\left(100 \mathrm{MHz}, \mathrm{CDCl}_{3}\right) \delta$ 156.7, 148.6, 139.1, 134.3, 134.0, 133.1, 129.6, 129.0, 125.6, 125.4, 121.1, 114.3, 60.7, 55.4, 53.7, 41.2, 31.6, 27.7, 26.6, 22.5, 14.0. IR (neat) 2927, 2855, 1666, 1505, 1462, 1395, 1351, 1242, 1210, 1090, $833 v_{\max } / \mathrm{cm}^{-1}$; MS (ESI) $\mathrm{m} / \mathrm{z}$ Calcd for $\mathrm{C}_{25} \mathrm{H}_{30} \mathrm{ClN}_{4} \mathrm{O}^{+}$: 437.2103 ; found: $437.2107[\mathrm{M}+\mathrm{H}]^{+}$. 
(E)-N-(7-(4-methoxybenzyl)-6-(4-methoxyphenyl)-7,8-dihydroimidazo[1,5-a]pyrazin-5(6H)-ylidene) cyclohexanamine (72). The crude material was purified by column chromatography ( $n$-hexane/ EtOAc 70:30) to give the product as yellowish oil (202 mg, 91\% yield). ${ }^{1} \mathrm{H}-\mathrm{NMR}\left(400 \mathrm{MHz}, \mathrm{CDCl}_{3}\right) \delta 8.46$ (s, $1 \mathrm{H}), 7.29(\mathrm{~d}, J=8.4 \mathrm{~Hz}, 2 \mathrm{H}), 7.15(\mathrm{~d}, J=8.4 \mathrm{~Hz}, 2 \mathrm{H}), 6.89(\mathrm{~d}, J=8.4 \mathrm{~Hz}, 2 \mathrm{H}), 6.84(\mathrm{~d}, J=8.4 \mathrm{~Hz}, 2 \mathrm{H})$, $6.74(\mathrm{~s}, 1 \mathrm{H}), 4.95(\mathrm{~s}, 1 \mathrm{H}), 3.82(\mathrm{~s}, 3 \mathrm{H}), 3.79(\mathrm{~s}, 3 \mathrm{H}), 3.77-3.73(\mathrm{~m}, 1 \mathrm{H}), 3.65-3.57(\mathrm{~m}, 3 \mathrm{H}), 3.13-3.07(\mathrm{~m}$, 1H), 1.74-1.01 (m, 10H); ${ }^{13} \mathrm{C}-\mathrm{NMR}\left(100 \mathrm{MHz}, \mathrm{CDCl}_{3}\right) \delta 159.5,159.1,145.8,132.8,130.0,129.6,129.3$, 126.8, 125.4, 124.8, 114.0, 113.9, 58.7, 57.4, 56.7, 55.2 (2C), 40.8, 34.1, 33.4, 25.4, 24.1, 24.0. IR (neat) 2928, $1676,1598,1509,1459,1345,1249,1201,1033,732,698 v_{\max } / \mathrm{cm}^{-1}$; MS (ESI) $\mathrm{m} / z$ Calcd for $\mathrm{C}_{27} \mathrm{H}_{33} \mathrm{~N}_{4} \mathrm{O}_{2}{ }^{+}$: 445.2599; found: $445.2600[\mathrm{M}+\mathrm{H}]^{+}$.

(E)-N-(7-(4-methoxybenzyl)-6-phenethyl-7,8-dihydroimidazo[1,5- $a$ ]pyrazin-5(6H)-ylidene) cyclohexanamine (73). The crude material was purified by column chromatography ( $n$-hexane/ EtOAc 70:30) to give the product as off-white solid (216 mg, 98\% yield). ${ }^{1} \mathrm{H}-\mathrm{NMR}\left(400 \mathrm{MHz}, \mathrm{CDCl}_{3}\right) \delta 8.31$ (s, $1 \mathrm{H}), 7.27-7.10(\mathrm{~m}, 7 \mathrm{H}), 6.84(\mathrm{~d}, J=8.4 \mathrm{~Hz}, 2 \mathrm{H}), 6.76(\mathrm{~s}, 1 \mathrm{H}), 4.14-4.10(\mathrm{~m}, 1 \mathrm{H}), 3.80(\mathrm{~s}, 3 \mathrm{H}), 3.81-3.72(\mathrm{~m}$, 2H), 3.59-3.43 (m, 2H), 2.95-2.70 (m, 3H), 2.17-2.13 (m, 1H), 1.73-1.00 (m, 11H) ${ }^{13} \mathrm{C}-\mathrm{NMR}(100 \mathrm{MHz}$, $\left.\mathrm{CDCl}_{3}\right) \delta 159.2,147.8,140.9,130.2,129.8,128.5(4 \mathrm{C}), 126.2,125.0,124.4,113.8,58.2,56.3,55.3,54.3,39.7$, 34.3, 34.0, 31.6, 31.4, 25.4, 24.2. IR (neat) 3022, 2933, 2858, 1661, 1513, 1467, 1349, 1252, 1220, 1037, 815 $v_{\max } / \mathrm{cm}^{-1}$; Mp 119-120 ${ }^{\circ} \mathrm{C}$; MS (ESI) $\mathrm{m} / z$ Calcd for $\mathrm{C}_{28} \mathrm{H}_{35} \mathrm{~N}_{4} \mathrm{O}^{+}$: 443.2806; found: $443.2805[\mathrm{M}+\mathrm{H}]^{+}$.

(E)-N-(7-(4-methoxybenzyl)-6-phenyl-7,8-dihydroimidazo[1,5- $a$ ]pyrazin-5(6H)-ylidene)-2,4,4trimethylpentan-2-amine (74). The crude material was purified by column chromatography ( $n$-hexane/ EtOAc 80:20) to give the product as colorless to yellowish oil (206 mg, 93\% yield). ${ }^{1} \mathrm{H}-\mathrm{NMR}$ $\left(400 \mathrm{MHz}, \mathrm{CDCl}_{3}\right) \delta 8.43(\mathrm{~s}, 1 \mathrm{H}), 7.33-7.19(\mathrm{~m}, 7 \mathrm{H}), 6.90(\mathrm{~d}, J=8.0 \mathrm{~Hz}, 2 \mathrm{H}), 6.73(\mathrm{~s}, 1 \mathrm{H}), 5.14(\mathrm{~s}, 1 \mathrm{H})$, $3.82(\mathrm{~s}, 3 \mathrm{H}), 3.73(\mathrm{~s}, 2 \mathrm{H}), 3.57\left(\mathrm{dd}, J_{a b}=16.4 \mathrm{~Hz}, 2 \mathrm{H}\right), 1.53\left(\mathrm{dd}, J_{a b}=14.4 \mathrm{~Hz}, 2 \mathrm{H}\right), 1.17(\mathrm{~s}, 3 \mathrm{H}), 1.03(\mathrm{~s}$, $3 \mathrm{H}), 0.95(\mathrm{~s}, 9 \mathrm{H}) ;{ }^{13} \mathrm{C}-\mathrm{NMR}\left(100 \mathrm{MHz}, \mathrm{CDCl}_{3}\right) \delta 159.1,142.0,135.6,129.8,129.7,128.7,128.6,128.2$, 125.7, 124.7, 113.9, 62.3, 58.7, 58.4, 57.5, 55.3, 40.7, 31.9, 31.8, 30.7. IR (neat) 2953, 1674, 1511, 1459, 1338, 1246, 1207, 1191, 699, $654 v_{\max } / \mathrm{cm}^{-1}$; MS (ESI) $\mathrm{m} / z$ Calcd for $\mathrm{C}_{28} \mathrm{H}_{37} \mathrm{~N}_{4} \mathrm{O}^{+}: 445.2962$; found: fragment ion (loss of 2,4,4-trimethyl-N-methylenepentan-2-amine moiety): $306.1602[\mathrm{M}+\mathrm{H}]^{+}$.

(E)-N-(7-(4-methoxybenzyl)-6-phenethyl-7,8-dihydroimidazo[1,5-a]pyrazin-5(6H)-ylidene)

naphthalen-1-amine (75). The crude material was purified by column chromatography ( $n$-hexane/ EtOAc 70:30) to give the product as yellowish solid (182 mg, 75\% yield). ${ }^{1} \mathrm{H}-\mathrm{NMR}\left(400 \mathrm{MHz}, \mathrm{CDCl}_{3}\right) \delta$ $8.47(\mathrm{~s}, 1 \mathrm{H}), 7.81(\mathrm{~d}, J=8.0 \mathrm{~Hz}, 1 \mathrm{H}), 7.72(\mathrm{~d}, J=8.0 \mathrm{~Hz}, 1 \mathrm{H}), 7.60(\mathrm{~d}, J=7.6 \mathrm{~Hz}, 1 \mathrm{H}), 7.48-7.42(\mathrm{~m}$, $2 \mathrm{H}), 7.26(\mathrm{~s}, 1 \mathrm{H}), 7.14(\mathrm{~d}, J=8.0 \mathrm{~Hz}, 2 \mathrm{H}), 6.96-6.59(\mathrm{~m}, 9 \mathrm{H}), 3.99\left(\mathrm{dd}, J_{a b}=16.8 \mathrm{~Hz}, 2 \mathrm{H}\right), 3.81(\mathrm{~s}, 3 \mathrm{H})$, $3.76-3.72(\mathrm{~m}, 1 \mathrm{H}), 3.61\left(\mathrm{dd}, J_{a b}=13.2 \mathrm{~Hz}, 2 \mathrm{H}\right), 2.68-2.48(\mathrm{~m}, 2 \mathrm{H}), 2.11-2.04(\mathrm{~m}, 1 \mathrm{H}), 1.85-1.76(\mathrm{~m}, 1 \mathrm{H})$; ${ }^{13} \mathrm{C}-\mathrm{NMR}\left(100 \mathrm{MHz}, \mathrm{CDCl}_{3}\right) \delta 159.1,151.9,144.1,139.8,134.0,133.4,130.7,129.9,129.3$ (2C), 128.0, $127.9,127.7,127.3,126.4,125.9,125.7,124.9,124.8,120.6,115.6,113.9,58.1,55.3,55.0,39.7,31.1,29.8$. IR (neat) 3052, 2954, 2937, 2846, 1667, 1510, 1356, 1246, 1208, 1031, $862 v_{\max } / \mathrm{cm}^{-1}$; Mp 136-137 ${ }^{\circ} \mathrm{C}$; MS (ESI) $\mathrm{m} / \mathrm{z}$ Calcd for $\mathrm{C}_{32} \mathrm{H}_{31} \mathrm{~N}_{4} \mathrm{O}^{+}$: 487.2493; found: $487.2498[\mathrm{M}+\mathrm{H}]^{+}$.

(E)-2-methyl- $N$-(6-phenethyl-7-phenyl-7,8-dihydroimidazo[1,5- $a$ ]pyrazin-5(6H)-ylidene)propan-2amine (76). The crude material was purified by column chromatography ( $n$-hexane/ EtOAc 70:30) to give the product as colorless to brownish oil ( $173 \mathrm{mg}$, $93 \%$ yield). ${ }^{1} \mathrm{H}-\mathrm{NMR}\left(400 \mathrm{MHz}, \mathrm{CDCl}_{3}\right) \delta 8.18$ (s, 1H), 7.31-7.15 (m, 7H), 6.93-6.87 (m, 4H), 4.72-4.69 (m, 1H), $4.58(\mathrm{~s}, 2 \mathrm{H}), 2.97-2.70(\mathrm{~m}, 2 \mathrm{H}), 2.31-1.97$ $(\mathrm{m}, 2 \mathrm{H}), 1.15(\mathrm{~s}, 9 \mathrm{H}) ;{ }^{13} \mathrm{C}-\mathrm{NMR}\left(100 \mathrm{MHz}, \mathrm{CDCl}_{3}\right) \delta 150.2,144.8,140.2,129.4,128.7,128.5,126.4,125.2$, 123.9, 121.6, 118.9, 58.2, 54.4, 38.9, 32.1, 31.4, 31.3. IR (neat) 2967, 2924, 1678, 1598, 1494, 1458, 1344, $1201,924,754,697 v_{\max } / \mathrm{cm}^{-1}$; MS (ESI) $\mathrm{m} / \mathrm{z}$ Calcd for $\mathrm{C}_{24} \mathrm{H}_{29} \mathrm{~N}_{4}{ }^{+}: 373.2387$; found: fragment ion (loss of 2-methyl-N-methylenepropan-2-amine): $290.1654[\mathrm{M}+\mathrm{H}]^{+}$.

\section{2-methyl- $N$-(6-pentyl-7-phenyl-7,8-dihydroimidazo[1,5-a]pyrazin-5(6H)-ylidene)propan-2-amine} (77). The crude material was purified by column chromatography ( $n$-hexane/ EtOAc 90:10) to give the product as yellowish oil $\left(160.5 \mathrm{mg}, 95 \%\right.$ yield). ${ }^{1} \mathrm{H}-\mathrm{NMR}\left(400 \mathrm{MHz}, \mathrm{CDCl}_{3}\right)$ main isomer $\delta 8.16(\mathrm{~s}, 1 \mathrm{H})$, 7.23-7.19 (m, 2H), 6.91-6.86 (m, 4H), 4.84-4.80 (m, 1H), $4.48\left(\mathrm{dd}, J_{\mathrm{ab}}=16.8 \mathrm{~Hz}, 2 \mathrm{H}\right), 1.96-1.87(\mathrm{~m}, 1 \mathrm{H})$, 
1.65-1.52 (m, 3H), 1.37-1.17 (m, 4H), $1.27(\mathrm{~s}, 9 \mathrm{H}), 0.86-0.83(\mathrm{~m}, 3 \mathrm{H}) ;{ }^{13} \mathrm{C}-\mathrm{NMR}\left(100 \mathrm{MHz}, \mathrm{CDCl}_{3}\right)$ main isomer $\delta 156.4$, 145.4, 140.6, 129.4, 127.1, 123.6, 121.3, 118.5, 59.1, 55.6, 38.7, 31.4, 31.3, 28.6, 25.9, 22.5, 13.9. IR (neat) 2959, 2930, 2870, 1672, 1493, 1456, 1361, 1201, 1103, 750, 695vmax $/ \mathrm{cm}^{-1}$; MS (ESI) m/z Calcd for $\mathrm{C}_{21} \mathrm{H}_{31} \mathrm{~N}_{4}{ }^{+}$: 339.2544; found: fragment ion (loss of 2-methyl-N-methylenepropan-2-amine): $256.1810[\mathrm{M}+\mathrm{H}]^{+}$.

$N$-(6-isobutyl-7-phenyl-7,8-dihydroimidazo[1,5-a]pyrazin-5(6H)-ylidene)cyclohexanamine (78). The crude material was purified by column chromatography (n-hexane/ EtOAc 90:10) to give the product as yellowish oil $\left(157.5 \mathrm{mg}, 90 \%\right.$ yield). ${ }^{1} \mathrm{H}-\mathrm{NMR}\left(400 \mathrm{MHz}, \mathrm{CDCl}_{3}\right)$ main isomer $\delta 8.22(\mathrm{~s}, 1 \mathrm{H})$, 7.22-7.18 (m, 2H), 6.89-6.84 (m, 4H), 4.91-4.87 (m, 1H), $4.51\left(\mathrm{dd}, J_{\mathrm{AB}}=16.8 \mathrm{~Hz}, 2 \mathrm{H}\right), 3.47-3.42(\mathrm{~m}, 1 \mathrm{H})$, 1.97-1.11 (m, 13H), 0.98-0.96 (m, 6H); ${ }^{13} \mathrm{C}-\mathrm{NMR}\left(100 \mathrm{MHz}, \mathrm{CDCl}_{3}\right)$ main isomer $\delta 174.4,149.9,133.0$, 129.4, 124.9, 123.9, 121.1, 117.7, 70.07, 53.6, 43.7, 39.3, 34.1, 33.1, 25.5, 24.5, 24.4, 23.6. IR (neat) 2928, 2855, 1671, 1598, 1497, 1463, 1354, 1216, 924, 754, $693 v_{\max } / \mathrm{cm}^{-1}$; MS (ESI) $m / z$ Calcd for $\mathrm{C}_{22} \mathrm{H}_{31} \mathrm{~N}_{4}{ }^{+}$: 351.2544; found: $351.2543[\mathrm{M}+\mathrm{H}]^{+}$.

(E)-N-(6-(4-chlorophenyl)-7-hexyl-7,8-dihydroimidazo[1,5-a]pyrazin-5(6H)-ylidene)-2-methylpropan2-amine (79). The crude material was purified by column chromatography ( $n$-hexane/ EtOAc 97:3) to give the product as an off-white solid (137 mg, 71\% yield). ${ }^{1} \mathrm{H}-\mathrm{NMR}\left(400 \mathrm{MHz}, \mathrm{CDCl}_{3}\right) \delta 8.35$ (s, $1 \mathrm{H}), 7.24(\mathrm{~d}, J=8.4 \mathrm{~Hz}, 2 \mathrm{H}), 7.15(\mathrm{~d}, J=8.4 \mathrm{~Hz}, 2 \mathrm{H}), 6.67(\mathrm{~s}, 1 \mathrm{H}), 5.05(\mathrm{~s}, 1 \mathrm{H}), 3.53\left(\mathrm{dd}, J_{a b}=16.8 \mathrm{~Hz}\right.$, 2H), 2.64-2.51 (m, 2H), 1.57-1.50 (m, 2H), 1.34-1.24 (m, 6H), $1.21(\mathrm{~s}, 9 \mathrm{H}), 0.88-0.85(\mathrm{~m}, 3 \mathrm{H}) ;{ }^{13} \mathrm{C}$ NMR $\left(100 \mathrm{MHz}_{,} \mathrm{CDCl}_{3}\right) \delta 143.4,134.5,134.0,133.0,129.9,128.7,125.4,124.8,62.7,54.6,53.8,40.5$, 31.6, 31.3, 28.1, 26.7, 22.6, 14.0. IR (neat) 2949, 2933, 2865, 2815, 1665, 1489, 1458, 1340, 1202, 1088, $1013 v_{\max } / \mathrm{cm}^{-1}$; Mp 108-109 ${ }^{\circ} \mathrm{C}$; MS (ESI) $\mathrm{m} / \mathrm{z}$ Calcd for $\mathrm{C}_{22} \mathrm{H}_{32} \mathrm{ClN}_{4}{ }^{+}$: 387.2310; found: fragment ion (loss of 2-methyl-N-methylenepropan-2-amine): $304.1577[\mathrm{M}+\mathrm{H}]^{+}$.

(E)-N-(6-(4-chlorophenyl)-7-(4-methoxybenzyl)-7,8-dihydroimidazo[1,5-a]pyrazin-5(6H)-ylidene) cyclohexanamine (80). The crude material was purified by column chromatography ( $n$-hexane/EtOAc 70:30) to give the product as amorphous solid (205 mg, 91.5\% yield). ${ }^{1} \mathrm{H}-\mathrm{NMR}\left(400 \mathrm{MHz}, \mathrm{CDCl}_{3}\right) \delta$ $8.46(\mathrm{~s}, 1 \mathrm{H}), 7.31-7.18(\mathrm{~m}, 6 \mathrm{H}), 6.89(\mathrm{dd}, J=8.4 \mathrm{~Hz}, 2 \mathrm{H}), 6.75(\mathrm{~s}, 1 \mathrm{H}), 4.93(\mathrm{~s}, 1 \mathrm{H}), 3.82(\mathrm{~s}, 3 \mathrm{H}), 3.77-3.59$ (m, 4H), 3.08-3.02 (m, 1H), 1.75-1.01 (m, 10H); ${ }^{13} \mathrm{C}$ NMR (100 MHz, CDCl $) \delta 159.2,144.9,134.2,133.7$, 130.0, 129.5, 129.3, 128.9, 125.1, 125.0 (2C), 114.0, 58.4, 57.6, 56.8, 55.3, 40.9 (2C), 34.1, 33.4, 25.4, 24.1, 24.0. IR (neat) $2929,2854,1669,1511,1463,1348,1247,1190,1090,815,731 v_{\mathrm{max}} / \mathrm{cm}^{-1}$; MS (ESI) $\mathrm{m} / \mathrm{z}$ Calcd for $\mathrm{C}_{26} \mathrm{H}_{30} \mathrm{ClN}_{4} \mathrm{O}^{+}$: 449.2103; found: $449.2106[\mathrm{M}+\mathrm{H}]^{+}$.

\section{Conclusions}

In conclusion, in this manuscript we reported the one-pot multicomponent synthesis of substituted imidazopyrazines, starting from simple and easily available building blocks. Three covalent bonds and one six-membered ring were formed with this transformation under very mild reaction conditions and without the need of metal catalysts or protecting groups. Furthermore, the good to excellent yields, the ease of performance, and the possibility to use two different regioisomeric imidazolemethanamines and a benzimidazolic congener, make this novel transformation a very powerful tool to simply generate complexity and diversity.

This work demonstrated how, with the judicious choice of components, it is still possible to discover new productive interrupted Ugi reactions, after fifty years from the first report [39]. Furthermore, the drug-like nature of these compounds, along with their novelty, and therefore lack of intellectual properties, makes them interesting probes for medicinal chemistry applications. Studies on this topic are in progress and will be reported in due course.

Supplementary Materials: The following are available online: copies of ${ }^{1} \mathrm{H}$ and ${ }^{13} \mathrm{C}$ spectra; $\mathrm{X}$-ray data for 79 (CIF file, CCDC number 1884698).

Author Contributions: Research Strategy and Idea, G.C.T. and M.G.; Writing, G.C.T., M.G., and E.N.; Experiments and Analyses, U.G., R.H., E.D.G. and S.P.; X-Ray Analysis, F.M. 
Funding: This research was funded by the Italian Association for Cancer Research (AIRC; MFAG 18793 to M.G.) and by the University of Piemonte Orientale (grant Ricerca locale di Ateneo 2016 to G.C.T.).

Conflicts of Interest: The authors declare no conflict of interest.

\section{References}

1. Liu, Y.; Zhang, S.; Lindsey, J.S. Total synthesis campaigns toward chlorophylls and related natural hydroporphyrins-Diverse macrocycles, unrealized opportunities. Nat. Prod. Rep. 2018, 35, 879-901. [CrossRef]

2. Eckert, R. Synergy Effects in the Chemical Synthesis and Extensions of. Multicomponent Reactions (MCRs) - The low energy way to ultra-short syntheses of tailor-made molecules. Molecules 2017. [CrossRef] [PubMed]

3. Clark, H. Green chemistry: Challenges and opportunities. Green Chem. 1999, 1, 1-8. [CrossRef]

4. Denmark, S.E. Organic Synthesis: Wherefrom and whither? (Some very personal reflections). Isr. J. Chem. 2018, 58, 61-72. [CrossRef] [PubMed]

5. Lygin, A.V.; De Meijere, A. Isocyanides in the synthesis of nitrogen heterocycles. Angew. Chem. Int. Ed. 2010, 49, 9094-9124. [CrossRef] [PubMed]

6. Rotstein, B.H.; Zaretsky, S.; Rai, V.; Yudin, A.K. Small heterocycles in multicomponent reactions. Chem. Rev. 2014, 114, 8323-8359. [CrossRef]

7. Dömling, A.; Wang, W.; Wang, K. Chemistry and biology of multicomponent reactions. Chem. Rev. 2012, 112, 3083-3135. [CrossRef]

8. Van der Heijden, G.; Ruijter, E.; Orru, R.V.A. Efficiency, diversity, and complexity with multicomponent reactions. Synlett 2013, 2013, 666-685.

9. Nenajdenko, V.G. (Ed.) Isocyanide Chemistry; Wiley-VCH: Weinheim, Germany, 2012.

10. Orru, R.V.A.; Ruijter, E. (Eds.) Synthesis of Heterocycles via Multicomponent Reactions I.; Springer: Berlin/Heidelberg, Germany, 2010.

11. Orru, R.V.A.; Ruijter, E. (Eds.) Synthesis of Heterocycles via Multicomponent Reactions II.; Springer: Berlin/Heidelberg, Germany, 2010.

12. Gulevich, A.V.; Zhdanko, A.G.; Orru, R.V.A.; Nenajdenko, V.G. Isocyanoacetate derivatives: Synthesis, reactivity, and application. Chem. Rev. 2010, 110, 5235-5331. [CrossRef]

13. Ugi, I.; Werner, B.; Domling, A. The Chemistry of isocyanides, their Multicomponent reactions and their libraries. Molecules 2003, 8, 53-66. [CrossRef]

14. Hulme, C.; Gore, V. "Multi-component reactions: Emerging chemistry in drug discovery" 'from xylocain to crixivan'. Curr. Med. Chem. 2003, 10, 51-80. [CrossRef] [PubMed]

15. Giustiniano, M.; Novellino, E.; Tron, G.C. Nitrile N-oxides and nitrile imines as new fuels for the discovery of novel isocyanide-based multicomponent reactions. Synthesis 2016, 48, 2721-2731. [CrossRef]

16. Wernerova, M.; Hudlicky, T. On the practical limits of determining isolated product yields and ratios of stereoisomers: Reflections, analysis, and redemption. Synlett 2010, 2701-2707.

17. Ugi, I.; Meyr, R.; Fetzer, U.; Steinbrückner, C. Versuche mit Isonitrilen. Angew. Chem. 1959, 71, 386-388.

18. Dömling, A.; Ugi, I. Multicomponent reactions with isocyanides. Angew. Chem. Int. Ed. 2000, 39, 3168-3210. [CrossRef]

19. Nenajdenko, V.G. Isocyanide Chemistry: Applications in Synthesis and Material Science; Wiley-VCH: Weinheim, Germany, 2012.

20. Giustiniano, M.; Moni, L.; Sangaletti, L.; Pelliccia, S.; Basso, A.; Novellino, E.; Tron, G.C. Interrupted Ugi and Passerini Reactions: An underexplored treasure Island. Synthesis 2018, 50, 3549-3570. [CrossRef]

21. Váradi, A.; Palmer, T.C.; Dardashti, R.N.; Majumdar, S. Isocyanide-based multicomponent reactions for the rynthesis of heterocycles. Molecules 2016. [CrossRef]

22. El Kaim, L.; Grimaud, L. Beyond the Ugi reaction: Less conventional interactions between isocyanides and iminium species. Tetrahedron 2009, 65, 2153-2171. [CrossRef]

23. Gesu, A.; Pozzoli, C.; Torre, E.; Aprile, S.; Pirali, T. Transition-metal-free synthesis of 2-arylimidazolones via cascade reaction between arynes and $\alpha, \alpha^{\prime}$-disubstituted $\alpha$-isocyanoacetamides. Org. Lett. 2016, 18, 1992-1995. [CrossRef] 
24. Groebke, K.; Weber, L.; Mehlin, F. Synthesis of imidazo[1,2-a] annulated pyridines, pyrazines and pyrimidines by a novel three-component condensation. Synlett 1998, 1998, 661-663. [CrossRef]

25. Blackburn, C.; Guan, B.; Fleming, P.; Shiosaki, K.; Tsai, S. Parallel Synthesis of 3-Aminoimidazo[1,2-a]pyridines and pyrazines by a new three-component condensation. Tetrahedron Lett. 1998, 39, 3635-3638. [CrossRef]

26. Bienaymé, H.; Bouzid, K.A. New heterocyclic multicomponent reaction for the combinatorial synthesis of fused 3-aminoimidazoles. Angew. Chem. Int. Ed. 1998, 37, 2234-2237. [CrossRef]

27. Martinez-Ariza, G.; Ayaz, M.; Medda, F.; Hulme, C. Synthesis of diverse nitrogen-enriched heterocyclic scaffolds using a suite of tunable one-pot multicomponent reactions. J. Org. Chem. 2014, 79, 5153-5162. [CrossRef]

28. Carballares, S.; Espinosa, J.F. Revision of outcome and mechanism of a new multicomponent reaction. Org. Lett. 2005, 7, 2329-2331. [CrossRef]

29. Boström, J.; Brown, D.G.; Young, R.J.; Keserü, G.M. Expanding the medicinal chemistry synthetic toolbox. Nat. Rev. Drug. Discov. 2018, 17, 709-727. [CrossRef]

30. Cerra, B.; Carotti, A.; Passeri, D.; Sardella, R.; Moroni, G.; Di Michele, A.; Macchiarulo, A.; Pellicciari, R.; Gioiello, A. Exploiting chemical toolboxes for the expedited generation of tetracyclic quinolines as a novel class of PXR agonists. ACS Med. Chem. Lett. 2019, 10, 677-681. [CrossRef]

31. Paganoa, N.; Terietea, P.; Mattmann, M.E.; Yang, L.; Snyder, B.A.; Cai, Z.; Heil, M.L.; Cosford, N.D.P. An integrated chemical biology approach reveals the mechanism of action of HIV replication inhibitors. Bioorg. Med. Chem. 2017, 25, 6248-6265. [CrossRef]

32. Reutlinger, M.; Rodrigues, T.; Schneider, P.; Schneider, G. Combining On-chip synthesis of a focused combinatorial library with computational target prediction reveals imidazopyridine GPCR ligands. Angew. Chem. Int. Ed. 2014, 53, 582-585. [CrossRef]

33. Farruja, L. ORTEP-3 for Windows-A version of ORTEP-III with a Graphical User Interface (GUI). J. Appl. Cryst. 1997, 30, 565. [CrossRef]

34. Cremer, D.; Pople, J.A. General definition of ring puckering coordinates. J. Am. Chem. Soc. 1975, 97, 1354-1358. [CrossRef]

35. Tron, G.C. Off the Beaten Track: The Use of Secondary Amines in the Ugi Reaction. Eur. J. Org. Chem. 2013, 2013, 1849-1859. [CrossRef]

36. Baldwin, J.E. Rules for ring closure. J. Chem. Soc. Chem. Commun. 1976. [CrossRef]

37. Hegarthy, A.F. Stereospecific reactions of nitrilium ions and analogous 1,3-dipoles. Acc. Chem. Res. 1980, 13, 448-453. [CrossRef]

38. Hegarty, A.F.; Tynan, N.M.; Fergus, S. Rate-determining nitrogen inversion in the isomerisation of isoimides to imides and azides to tetrazoles: Direct observation of intermediates stabilized by trifluoroethyl groups. J. Chem. Soc. Perkin Trans. 2 2002, 7, 1328-1334. [CrossRef]

39. Deyrup, J.A.; Vestling, M.M.; Hagan, W.V.; Yun, H.Y. Reactions of imines with t-butyl isocyanide. Tetrahedron 1969, 25, 1467-1478. [CrossRef]

Sample Availability: All data generated and/or compound samples analyzed during this study are included in this article and are available from the corresponding authors on reasonable request.

(C) 2019 by the authors. Licensee MDPI, Basel, Switzerland. This article is an open access article distributed under the terms and conditions of the Creative Commons Attribution (CC BY) license (http://creativecommons.org/licenses/by/4.0/). 